\title{
A recursive multibody formalism for systems with small mass and inertia terms
}

\author{
M. Arnold \\ Martin Luther University Halle-Wittenberg, NWF II - Institute of Mathematics, 06099 Halle (Saale), Germany
}

Correspondence to: M. Arnold (martin.arnold@mathematik.uni-halle.de)

Received: 18 November 2012 - Revised: 14 February 2013 - Accepted: 7 March 2013 - Published: 17 May 2013

\begin{abstract}
Complex multibody system models that contain bodies with small mass or nearly singular inertia tensor may suffer from high frequency solution components that deteriorate the solver efficiency in time integration. Singular perturbation theory suggests to neglect these small mass and inertia terms to allow a more efficient computation of the smooth solution components. In the present paper, a recursive multibody formalism is developed to evaluate the equations of motion for a tree structured $N$ body system with $O(N)$ complexity even if isolated bodies have a rank-deficient body mass matrix. The approach is illustrated by some academic test problems in 2-D.
\end{abstract}

1

\section{Introduction}

Classical time integration methods in technical simulation are tailored to problems with smooth solution. Small system parameters in a mathematical model may introduce rapidly oscillating or strongly damped solution components that cause problems in time integration. Singular perturbation theory gives much insight in the analytical background of these phenomena and allows furthermore an efficient approximation of smooth solutions neglecting all terms that contain small parameters, see, e.g., Hairer and Wanner (1996).

The application of these classical results to multibody dynamics is non-trivial since the numerical algorithms for evaluating the equations of motion efficiently (multibody formalisms) are based on regularity assumptions that may be violated if small mass and inertia terms are neglected. A modified multibody formalism for chain structured multibody systems with an isolated "zero mass" body was developed in Arnold et al. (2010).

The present paper is a revised and extended version of the author's contribution to this conference paper. A recursive multibody formalism is developed for tree structured systems with bodies that suffer from a rank-deficient body mass matrix and may be considered as limit case of systems with bodies of (very) small mass or nearly singular inertia tensor. This research is guided by well known results from general singu- lar perturbation theory, see Hairer and Wanner (1996), and its extensions to singularly perturbed problems in the context of multibody dynamics by Lubich (1993) and Stumpp (2008).

Related problems are, e.g., the modelling of serial springdamper elements using an auxiliary zero mass body between spring and damper (Eich-Soellner and Führer, 1998, Section 1.3.4), the modification of inertia forces for highfrequency eigenmodes of flexible bodies in multibody system models for the analysis of elastohydrodynamic bearing coupling in Schönen (2003) and recently proposed methods from FE contact mechanics in Hager and Wohlmuth (2009).

For real-time applications in multibody dynamics, the neglection of inertia forces for small mass bodies was studied by Eichberger and Rulka (2004). A more detailed analysis shows, that this neglection of inertia forces is straightforward if all small mass bodies of the multibody system are leaf bodies in the kinematic tree. In numerical experiments for the model of a walking mobile robot (mobot) with stiff contact forces between lightweight legs and ground floor, the numerical effort was reduced by a factor of 4 , see Weber et al. (2012).

The singular perturbation analysis is technically more complicated for multibody system models with small mass bodies having in the kinematic tree a successor of substantially larger mass since classical multibody formalisms and topological solvers are not applicable to the limit case of zero 
mass bodies in a kinematic chain. In Burgermeister et al. (2011), a smoothed velocity approximation for small mass bodies was proposed as a work-around.

In the present paper, we discuss an alternative approach that extends the recursive multibody formalism directly to kinematic trees containing bodies of vanishing mass or rankdeficient inertia tensor. The classical set of second order equations of motion in the joint coordinates $\boldsymbol{q}(t)$ is substituted by a suitable combination of second and first order ODEs describing the system dynamics in the limit case of a zero mass body. These results extend the previous analysis for chain structured systems in Arnold et al. (2010) and may be considered as a next step to extend advanced multibody formalisms for flexible multibody systems to models with bodies of (very) small mass.

The remaining part of the paper is organized as follows: Basic results of singular perturbation theory and its application to multibody system dynamics are recalled in Sect. 2. A recursive multibody formalism and the resulting mixed set of first and second order equations of motion for a body of rankdeficient body mass matrix are derived in Sect. 3. Finally, two simple test problems are discussed in Sect. 4. Some basic information on Moore-Penrose pseudo-inverses is provided in Appendix A.

\section{Singular perturbations in multibody system models}

After a short introduction to singularly perturbed ODEs we consider in the present section singular perturbations in multibody system models that are caused by stiff potential forces, see Sect. 2.1. Furthermore, some typical problems in the dynamical simulation of multibody system models with small mass bodies are illustrated by the analysis of two coupled oscillators in Sect. 2.2.

\subsection{Time integration of singularly perturbed problems in multibody dynamics}

The generic form of singularly perturbed ODEs are partitioned systems

$$
\begin{aligned}
\dot{y}_{\varepsilon} & =\varphi\left(y_{\varepsilon}, z_{\varepsilon}\right), \\
\varepsilon \dot{z}_{\varepsilon} & =\gamma\left(y_{\varepsilon}, z_{\varepsilon}\right)
\end{aligned}
$$

with a small perturbation parameter $\varepsilon>0$ that are considered at a finite time interval $\left[0, t_{\mathrm{e}}\right]$, see Hairer and Wanner (1996, Chapter VI) and the references therein.

For any given initial value $\boldsymbol{y}^{0}$, the singularly perturbed system (1) has a smooth solution $\left(\boldsymbol{y}_{\varepsilon}^{0}(t), \boldsymbol{z}_{\varepsilon}^{0}(t)\right)$ with $\boldsymbol{y}_{\varepsilon}^{0}(0)=\boldsymbol{y}^{0}$ if the right hand side of the singularly perturbed subsystem (1b) has a Jacobian with eigenvalues satisfying along each solution trajectory $\left(\boldsymbol{y}_{\varepsilon}(t), \boldsymbol{z}_{\varepsilon}(t)\right)$ the condition

$\operatorname{Re} \lambda_{i}\left[\left(\partial \gamma / \partial z_{\varepsilon}\right)\left(y_{\varepsilon}, z_{\varepsilon}\right)\right] \leq-\beta<0$ for some positive constant $\beta>0$. This smooth solution remains in an $O(\varepsilon)$-neighbourhood of the solution $\left(y_{0}(t), z_{0}(t)\right)$ of the reduced problem that results from setting formally the perturbation parameter in (1) to $\varepsilon:=0$. We get

$$
\begin{aligned}
\dot{y}_{0} & =\varphi\left(y_{0}, z_{0}\right), \\
\mathbf{0} & =\gamma\left(y_{0}, z_{0}\right)
\end{aligned}
$$

with $y_{0}(0)=y^{0}$ and $z_{0}(0)$ being implicitly defined by (2b). The general solution of the singularly perturbed problem (1) has the form

$$
\begin{aligned}
& \boldsymbol{y}_{\varepsilon}(t)=\boldsymbol{y}_{\varepsilon}^{0}(t)+\varepsilon \boldsymbol{\eta}_{\varepsilon}(t / \varepsilon)=\boldsymbol{y}_{0}(t)+O(\varepsilon), \\
& \boldsymbol{z}_{\varepsilon}(t)=z_{\varepsilon}^{0}(t)+\zeta_{\varepsilon}(t / \varepsilon)=z_{0}(t)+O(\varepsilon)+\zeta_{\varepsilon}(t / \varepsilon)
\end{aligned}
$$

with smooth functions $\boldsymbol{\eta}_{\varepsilon}(t / \varepsilon), \zeta_{\varepsilon}(t / \varepsilon)$ that decay like $\exp (-\bar{\beta} t / \varepsilon)$ for some positive constant $\bar{\beta} \in(0, \beta)$, see Hairer and Wanner (1996, Theorem VI.3.2).

For many stiff integrators, the numerical solution of (1) may be decomposed as well in a smooth part and a rapidly decaying part reflecting the transient behaviour for initial values $\left(\boldsymbol{y}_{\varepsilon}(0), \boldsymbol{z}_{\varepsilon}(0)\right)$ that do not belong to a smooth solution. If there is no particular interest in this transient phase, an approximate numerical solution may be obtained much more efficiently solving for given initial values $\boldsymbol{y}_{0}(0):=\boldsymbol{y}_{\varepsilon}(0)$ the DAE (2) by appropriate time integration methods (Hairer and Wanner, 1996, Chapter VI). Note, that the initial values $z_{0}(0)$ in DAE (2) are not free but have to satisfy the consistency condition $\boldsymbol{\gamma}\left(\boldsymbol{y}_{0}(0), z_{0}(0)\right)=\mathbf{0}$.

Lubich (1993) extended these classical results to a class of singular singularly perturbed problems

$$
\mathbf{M}(\boldsymbol{q}) \ddot{\boldsymbol{q}}=\psi(\boldsymbol{q}, \dot{\boldsymbol{q}})-\frac{1}{\varepsilon^{2}} \nabla U(\boldsymbol{q})
$$

with $\boldsymbol{q}(t)$ denoting the position coordinates of a multibody system. Matrix $\mathbf{M}(\boldsymbol{q})$ is the symmetric positive definite mass matrix and $\boldsymbol{\psi}(\boldsymbol{q}, \dot{\boldsymbol{q}})$ denotes a vector of forces and momenta. The crucial term in (4) are the (very) stiff potential forces $-\varepsilon^{-2} \nabla U(\boldsymbol{q})$ that depend on the perturbation parameter $\varepsilon$ with $0<\varepsilon \ll 1$. If the potential $U(\boldsymbol{q})$ attains a local minimum on a manifold $\mathcal{U}$ and is strongly convex along all directions that are non-tangential to $\mathcal{U}$, then the smooth solution of (4) may be approximated up to $O\left(\varepsilon^{2}\right)$ by the solution $\boldsymbol{q}_{0}(t)$ of a constrained (differential-algebraic) system with $\boldsymbol{q}_{0}(t) \in \mathcal{U}$, $(t \geq 0)$. In general, this constrained system may be solved much more efficiently than the original singularly perturbed problem (4), see Hairer and Wanner (1996).

In Stumpp (2008), these results were extended to mechanical systems with strong damping forces $-\varepsilon^{-1} \mathbf{D}(\boldsymbol{q}) \dot{\boldsymbol{q}}$ in the right hand side of (4). In the limit case $\varepsilon \rightarrow 0$, the solution of the singularly perturbed problem is again approximated by the solution of a DAE problem that can be obtained in a robust and efficient way by standard DAE time integration methods, see Hairer and Wanner (1996). 


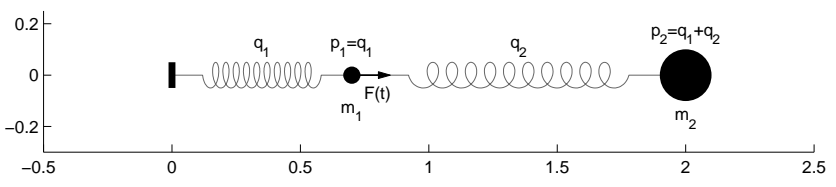

Figure 1. Example problem: two coupled oscillators with a fast oscillating small mass $m_{1}$, see Burgermeister et al. (2011).

\subsection{The small mass oscillator as singularly perturbed problem}

The eigenfrequency of a harmonic oscillator is given by $\omega=\sqrt{c / m}$ with mass $m$ and spring constant $c$. High frequency oscillations in a mechanical system may not only be introduced by (very) stiff potential forces but also by potential forces of moderate size that act on a body with (very) small mass.

In Burgermeister et al. (2011), this phenomenon was studied for the simple model problem in Fig. 1. In two coupled oscillators, a small mass $m_{1}$ is connected to a large mass $m_{2}$ and the reference system by stiff springs with constants $c_{1}, c_{2}$ and damping with damping parameters $d_{1}, d_{2}$. Both bodies can only move along the $\mathrm{x}$-axis. Additional forces $F(t)$ are only acting on $m_{1}$. In absolute coordinates $\boldsymbol{p}(t)=\left(p_{1}(t), p_{2}(t)\right)^{\top}$, the equations of motion are given by

$$
\begin{aligned}
m_{1} \ddot{p}_{1}=F(t)-d_{1} \dot{p}_{1} & -c_{1} p_{1} \\
& +d_{2}\left(\dot{p}_{2}-\dot{p}_{1}\right)+c_{2}\left(p_{2}-p_{1}\right),
\end{aligned}
$$$$
m_{2} \ddot{p}_{2}=-d_{2}\left(\dot{p}_{2}-\dot{p}_{1}\right)-c_{2}\left(p_{2}-p_{1}\right) \text {. }
$$

The small mass can oscillate very fast depending on the ratio of the masses and spring parameters. If the perturbation parameter $\varepsilon:=m_{1}$ gets smaller, the frequency of the oscillations increases and a time integration method with stepsize control would choose very small stepsizes to resolve these oscillations and meet the integration tolerances. The number of time steps and the computing time increase significantly, see Fig. 2. In the limit case $\varepsilon=m_{1}=0$, the inertia forces of the first mass point are neglected and (5a) results in an implicit first order differential equation

$0=F(t)-d_{1} \dot{p}_{1}-c_{1} p_{1}+d_{2}\left(\dot{p}_{2}-\dot{p}_{1}\right)+c_{2}\left(p_{2}-p_{1}\right)$.

The fast oscillations of the small mass disappear and the integrator can use large stepsizes, see Fig. 2.

In a system description by relative coordinates $\boldsymbol{q}(t)=\left(q_{1}(t), q_{2}(t)\right)^{\top}$ with $p_{1}(t)=: q_{1}(t)$ and $p_{2}(t)-p_{1}(t)$ $=: q_{2}(t)$, the limit process $\varepsilon \rightarrow 0$ causes substantial problems since the equations of motion (5b) of the large mass depend on $\ddot{p}_{2}=\ddot{q}_{1}+\ddot{q}_{2}$ but $\ddot{q}_{1}=\ddot{p}_{1}$ does not appear in (6). Furthermore, the differentiation of (6) w.r.t. time $t$ shows that $\ddot{q}_{1}(t)$ depends on the time derivative of $F(t)$ in the limit case $\varepsilon=m_{1}=0$.

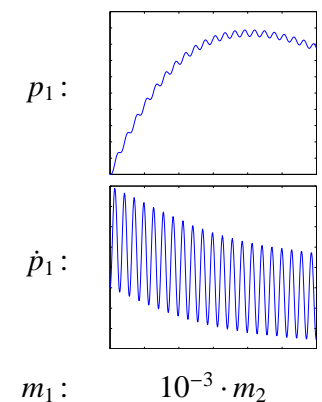

steps:
$10^{-3} \cdot m$
496

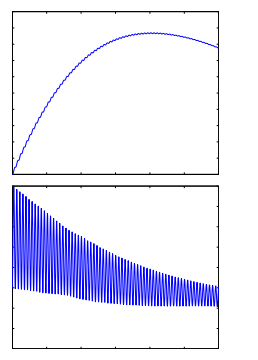

$10^{-4} \cdot m_{2}$

1489

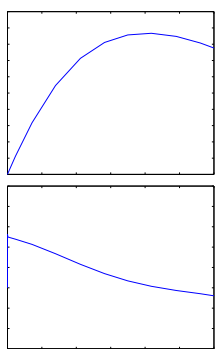

0

13
Figure 2. Oscillations and number of time steps for decreasing mass ratios $m_{1} / m_{2}$ (BDF solver DASSL with tolerances $10^{-6}$ for absolute and relative errors), see Burgermeister et al. (2011).

\section{Mixed coordinate formulation of the equations of motion}

(Very) small masses or (nearly) singular inertia tensors in a multibody system model may be interpreted as small perturbations. The analysis of Sect. 2.1 suggests to study a reduced system that neglects these perturbations. Therefore, we consider now the limit case of multibody systems that have one or more bodies with zero mass or singular inertia tensor, i.e., with a rank-deficient body mass matrix.

In that case, classical recursive multibody formalisms (“ $O(N)$-formalisms") like the ones by Brandl et al. (1988), Lubich et al. (1992) and Eichberger (1994) fail since they use the inverse of projected body mass matrices. Udwadia and Phohomsiri (2006) consider multibody systems with singular mass matrix but do not exploit the system's topology to evaluate the equations of motion efficiently by a sequence of forward and backward recursions in the kinematic tree.

In this section, we describe the multibody system by the absolute position and orientation of all $N$ bodies relative to the inertial frame and use joint coordinates as generalized coordinates for a tree structured system ( "mixed coordinates"), see also Schiehlen (1997). As in Lubich et al. (1992) and Eich-Soellner and Führer (1998), the recursive multibody formalism is interpreted as a block Gaussian elimination for a sparse, (very) large block-structured system of linear equations with the block structure being determined by the topology of the multibody system.

Using generalized inverses, the recursive formalism of Lubich et al. (1992) is modified to skip bodies with rankdeficient body mass matrix in the second forward recursion, see Sects. 3.2 and 3.3. The resulting equations of motion are not longer explicit but form a (linearly) implicit set of first and second order differential equations (Sect. 3.4). Finally, we show in Sect. 3.5 by some analytical transformations that the equations of motion form a first order index-1 DAE if a certain regularity assumption is satisfied. 


\subsection{Tree structured multibody systems: kinematics}

Recursive multibody formalisms are tailored to tree structured systems. Here, the term tree structure corresponds to the structure of the labelled graph being associated to the multibody system model. In this graph, each (rigid or flexible) body of the system is represented by a vertex. Two vertices of the graph are connected by an edge if and only if the corresponding bodies in the multibody system model are connected by a joint restricting their relative motion.

The graph of a tree structured multibody system is acyclic, i.e., it is free of loops. Then, the multibody system has a root body $(\bullet)^{(0)}$ that corresponds to the root vertex of the tree structured graph and is supposed to be inertially fixed. All other bodies $(\bullet)^{(i)}$ have a uniquely defined predecessor $(\bullet)^{\left(\pi_{i}\right)}$ in the kinematic tree. Each body $(\bullet)^{(i)}$ may have successors $(\bullet)^{(j)}$ being characterized by $\pi_{j}=i$ or, equivalently, by $j \in I_{i}:=\left\{k: \pi_{k}=i\right\}$ with an index set $I_{i}$ that represents the set of all successors of a given body $(\bullet)^{(i)}$ in the multibody system model. Bodies without successors $\left(I_{i}=\emptyset\right)$ correspond to leafs of the kinematic tree and are therefore called "leaf bodies".

We suppose that position and orientation of body $(\bullet)^{(i)}$ may be characterized by (absolute) position coordinates $\boldsymbol{p}_{i}(t) \in \mathbb{R}^{d}$ with $d=6$ for 3-D models and $d=3$ for 2-D models (the position of point masses may be characterized by $d=3$ absolute coordinates in 3-D and by $d=2$ absolute coordinates in 2 -D, see Sect. 4 below). The relative position and orientation of body $(\bullet)^{(i)}$ W.r.t. its predecessor $(\bullet)^{\left(\pi_{i}\right)}$ is characterized by joint coordinates $\boldsymbol{q}_{i}(t) \in \mathbb{R}^{n_{i}}$ representing the $n_{i}$ degrees of freedom of the joint connecting $(\bullet)^{(i)}$ with $(\bullet)^{\left(\pi_{i}\right)}$ :

$\mathbf{0}=\boldsymbol{k}_{i}\left(\boldsymbol{p}_{i}, \boldsymbol{p}_{\pi_{i}}, \boldsymbol{q}_{i}, t\right)$

Here and in the following we suppose that (7) is locally uniquely solvable w.r.t. $\boldsymbol{p}_{i}$ and that the Jacobian $\mathbf{K}_{i}=\partial \boldsymbol{k}_{i} / \partial \boldsymbol{p}_{i}$ is non-singular along the solution. In its most simple form, Eq. (7) defines $\boldsymbol{p}_{i}$ explicitly by $\boldsymbol{p}_{i}(t)=\boldsymbol{r}_{i}\left(\boldsymbol{p}_{\pi_{i}}(t), \boldsymbol{q}_{i}(t), t\right)$ resulting in $\mathbf{K}_{i}=\mathbf{I}_{d}$.

The kinematic relations (7) at the level of position coordinates imply relations at the level of velocity and acceleration coordinates that may formally be obtained by (total) differentiation of (7) w.r.t. time $t$ :

$$
\begin{aligned}
\mathbf{0} & =\frac{\mathrm{d}}{\mathrm{d} t} \boldsymbol{k}_{i}\left(\boldsymbol{p}_{i}(t), \boldsymbol{p}_{\pi_{i}}(t), \boldsymbol{q}_{i}(t), t\right) \\
& =\mathbf{K}_{i} \dot{\boldsymbol{p}}_{i}+\mathbf{H}_{i} \dot{\boldsymbol{p}}_{\pi_{i}}+\mathbf{J}_{i} \dot{\boldsymbol{q}}_{i}+\boldsymbol{k}_{i}^{(\mathrm{I})}\left(\boldsymbol{p}_{0}, \boldsymbol{p}, \boldsymbol{q}, t\right), \\
\mathbf{0} & =\mathbf{K}_{i} \ddot{\boldsymbol{p}}_{i}+\mathbf{H}_{i} \ddot{\boldsymbol{p}}_{\pi_{i}}+\mathbf{J}_{i} \ddot{\boldsymbol{q}}_{i}+\boldsymbol{k}_{i}^{(\mathrm{II})}\left(\boldsymbol{p}_{0}, \dot{\boldsymbol{p}}_{0}, \boldsymbol{p}, \dot{\boldsymbol{p}}, \boldsymbol{q}, \dot{\boldsymbol{q}}, t\right)
\end{aligned}
$$

with

$\mathbf{K}_{i}:=\frac{\partial \boldsymbol{k}_{i}}{\partial \boldsymbol{p}_{i}} \in \mathbb{R}^{d \times d}, \quad \mathbf{H}_{i}:=\frac{\partial \boldsymbol{k}_{i}}{\partial \boldsymbol{p}_{\pi_{i}}} \in \mathbb{R}^{d \times d}, \quad \mathbf{J}_{i}:=\frac{\partial \boldsymbol{k}_{i}}{\partial \boldsymbol{q}_{i}} \in \mathbb{R}^{d \times n_{i}}$

It is supposed that the joint coordinates $\boldsymbol{q}_{i}(t)$ are defined such that all Jacobians $\mathbf{J}_{i}$ have full column rank: $\operatorname{rank} \mathbf{J}_{i}=n_{i} \leq d$.
Functions $\boldsymbol{k}_{i}^{(\mathrm{I})}:=\partial \boldsymbol{k}_{i} / \partial t$ and $\boldsymbol{k}_{i}^{(\mathrm{II})}$ summarize partial time derivatives and all lower order terms in the first and second time derivative of (7), respectively. They may depend on the (absolute) coordinates $\boldsymbol{p}_{0}$ of the root body, on the absolute coordinates $\boldsymbol{p}:=\left(\boldsymbol{p}_{1}, \ldots, \boldsymbol{p}_{N}\right)$ of the remaining $N$ bodies in the system, on the corresponding joint coordinates $\boldsymbol{q}:=\left(\boldsymbol{q}_{1}, \ldots, \boldsymbol{q}_{N}\right)$ and on $\dot{\boldsymbol{p}}_{0}, \dot{\boldsymbol{p}}$ and $\dot{\boldsymbol{q}}$.

Note, that $(9)$ is simplified substantially for all bodies $(\bullet)^{(i)}$ that follow directly the root body $\left(\pi_{i}=0\right)$ since the root body is inertially fixed $\left(\ddot{\boldsymbol{p}}_{\pi_{i}}=\mathbf{0}\right)$ :

$\mathbf{0}=\mathbf{K}_{i} \ddot{\boldsymbol{p}}_{i}+\mathbf{J}_{i} \ddot{\boldsymbol{q}}_{i}+\boldsymbol{k}_{i}^{(\mathrm{II})}\left(\boldsymbol{p}_{0}, \dot{\boldsymbol{p}}_{0}, \boldsymbol{p}, \dot{\boldsymbol{p}}, \boldsymbol{q}, \dot{\boldsymbol{q}}, t\right) \quad$ if $\quad \pi_{i}=0$.

In recursive multibody formalisms, it is supposed that position and velocity of the root body $\left(\boldsymbol{p}_{0}(t), \dot{\boldsymbol{p}}_{0}(t)\right)$ and all joint coordinates $\boldsymbol{q}_{i}(t), \dot{\boldsymbol{q}}_{i}(t),(i=1, \ldots, N)$, at a current time $t$ are known. Starting from the root body, the absolute position and velocity coordinates $\boldsymbol{p}_{i}(t), \dot{\boldsymbol{p}}_{i}(t)$ of all $N$ bodies $(\bullet)^{(i)}$, $(i=1, \ldots, N)$, may then be computed recursively using (7) and (8), respectively, (forward recursion).

\subsection{Tree structured multibody systems: equilibrium conditions}

The equations of motion of a multibody system with $N$ bodies may be obtained from the equilibrium conditions for forces and momenta for each individual body that are formulated in absolute coordinates $\boldsymbol{p}_{i}$ :

$\mathbf{M}_{i} \ddot{\boldsymbol{p}}_{i}+\mathbf{K}_{i}^{\top} \boldsymbol{\mu}_{i}+\sum_{j \in I_{i}} \mathbf{H}_{j}^{\top} \boldsymbol{\mu}_{j}=\boldsymbol{f}_{i},(i=1, \ldots, N)$.

The body mass matrix $\mathbf{M}_{i} \in \mathbb{R}^{d \times d}$ contains mass and inertia tensor of body $(\bullet)^{(i)}$ and is supposed to be symmetric, positive semi-definite. The equilibrium conditions contain the reaction forces of the joints connecting body $(\bullet)^{(i)}$ with its predecessor $\left(\mathbf{K}_{i}^{\top} \boldsymbol{\mu}_{i}\right)$ and with its successors in the kinematic tree $\left(\mathbf{H}_{j}^{\top} \boldsymbol{\mu}_{j}, j \in I_{i}\right)$. All remaining forces and momenta acting on body $(\bullet)^{(i)}$ are summarized in the force vector $\boldsymbol{f}_{i}=\boldsymbol{f}_{i}(\boldsymbol{p}, \dot{\boldsymbol{p}}, \boldsymbol{q}, \dot{\boldsymbol{q}}, t) \in \mathbb{R}^{d}$.

The specific structure of the joint reaction forces with Lagrange multipliers $\boldsymbol{\mu}_{i}(t) \in \mathbb{R}^{d}$ that satisfy

$\mathbf{J}_{i}^{\top} \boldsymbol{\mu}_{i}=\mathbf{0},(i=1, \ldots, N)$,

results from the joint equations (7) and from d'Alembert's principle since the virtual work of constraint forces vanishes for all (virtual) displacements being compatible with (7). In (12), matrix $\mathbf{J}_{i}$ denotes the Jacobian of the constraint function $\boldsymbol{k}_{i}$ w.r.t. joint coordinates $\boldsymbol{q}_{i} \in \mathbb{R}^{n_{i}}$, see Sect. 3.1 above.

For leaf bodies $(\bullet)^{(i)}$, the equilibrium conditions (11) get a simpler form since $I_{i}=\left\{j: \pi_{j}=i\right\}=\emptyset$. We obtain

$\overline{\mathbf{M}}_{i} \mathbf{K}_{i} \ddot{\boldsymbol{p}}_{i}+\boldsymbol{\mu}_{i}=\overline{\boldsymbol{f}}_{i}$

with $\overline{\boldsymbol{f}}_{i}:=\mathbf{K}_{i}^{-\top} \boldsymbol{f}_{i}, \mathbf{K}_{i}^{-\top}:=\left(\mathbf{K}_{i}^{\top}\right)^{-1}$ and the symmetric, positive semi-definite mass matrix $\overline{\mathbf{M}}_{i}:=\mathbf{K}_{i}^{-\top} \mathbf{M}_{i} \mathbf{K}_{i}^{-1}$. 
One of the basic components of recursive multibody formalisms are algorithms to transform the equilibrium conditions (11) recursively for all bodies $(\bullet)^{(i)}$ to the simpler form (13) with suitable $\overline{\mathbf{M}}_{i}$ and $\overline{\boldsymbol{f}}_{i}$. With the common assumption that all body mass matrices $\mathbf{M}_{i}$ are non-singular, $\boldsymbol{\mu}_{i}$ may be expressed in terms of $\ddot{\boldsymbol{p}}_{\pi_{i}}, \overline{\mathbf{M}}_{i}, \overline{\boldsymbol{f}}_{i}$ and $\boldsymbol{k}_{i}^{(\mathrm{II})}$ by block Gaussian elimination applied to (9), (12) and (13), see, e.g., Lubich et al. (1992).

It is an important observation that this backward recursion may be generalized to multibody systems with rankdeficient body mass matrices $\mathbf{M}_{i}$ as long as all body mass matrices $\mathbf{M}_{i}$ are symmetric, positive semi-definite. In the following, this will be shown by mathematical induction: let us suppose that the equilibrium conditions of all successors $(\bullet)^{(j)}$ of body $(\bullet)^{(i)}$ are given in form (13), i.e.,

$\overline{\mathbf{M}}_{j} \mathbf{K}_{j} \ddot{\boldsymbol{p}}_{j}+\boldsymbol{\mu}_{j}=\overline{\boldsymbol{f}}_{j},\left(j \in I_{i}\right)$.

Since $\boldsymbol{\mu}_{j}$ belongs to the null space of $\mathbf{J}_{j}^{\top}$, see (12), we get

$$
\begin{aligned}
\boldsymbol{\mu}_{j} & =\left(\mathbf{I}_{d}-\overline{\mathbf{M}}_{j} \mathbf{J}_{j}\left(\mathbf{J}_{j}^{\top} \overline{\mathbf{M}}_{j} \mathbf{J}_{j}\right)^{+} \mathbf{J}_{j}^{\top}\right) \boldsymbol{\mu}_{j} \\
& =\left(\mathbf{I}_{d}-\overline{\mathbf{M}}_{j} \mathbf{J}_{j}\left(\mathbf{J}_{j}^{\top} \overline{\mathbf{M}}_{j} \mathbf{J}_{j}\right)^{+} \mathbf{J}_{j}^{\top}\right)\left(\overline{\boldsymbol{f}}_{j}-\overline{\mathbf{M}}_{j} \mathbf{K}_{j} \ddot{\boldsymbol{p}}_{j}\right)
\end{aligned}
$$

with the Moore-Penrose pseudo-inverse $\left(\mathbf{J}_{j}^{\top} \overline{\mathbf{M}}_{j} \mathbf{J}_{j}\right)^{+}$of the projected body mass matrix $\mathbf{J}_{j}^{\top} \overline{\mathbf{M}}_{j} \mathbf{J}_{j} \in \mathbb{R}^{n_{j} \times n_{j}}$, see Remark 1 in Appendix A. Because of $\pi_{j}=i$, the term $\mathbf{K}_{j} \ddot{\boldsymbol{p}}_{j}$ is given by

$\mathbf{K}_{j} \ddot{\boldsymbol{p}}_{j}=-\mathbf{H}_{j} \ddot{\boldsymbol{p}}_{i}-\mathbf{J}_{j} \ddot{\boldsymbol{q}}_{j}-\boldsymbol{k}_{j}^{(\mathrm{II})}$,

see (9), and we obtain finally

$$
\begin{aligned}
\boldsymbol{\mu}_{j}=\left(\overline{\mathbf{M}}_{j}-\overline{\mathbf{M}}_{j} \mathbf{J}_{j}\left(\mathbf{J}_{j}^{\top} \overline{\mathbf{M}}_{j} \mathbf{J}_{j}\right)^{+} \mathbf{J}_{j}^{\top} \overline{\mathbf{M}}_{j}\right) \mathbf{H}_{j} \ddot{p}_{i} \\
+\left(\mathbf{I}_{d}-\overline{\mathbf{M}}_{j} \mathbf{J}_{j}\left(\mathbf{J}_{j}^{\top} \overline{\mathbf{M}}_{j} \mathbf{J}_{j}\right)^{+} \mathbf{J}_{j}^{\top}\right)\left(\overline{\boldsymbol{f}}_{j}+\overline{\mathbf{M}}_{j} \boldsymbol{k}_{j}^{(\mathrm{II})}\right)
\end{aligned}
$$

since

$$
\begin{aligned}
& \left(\overline{\mathbf{M}}_{j}-\overline{\mathbf{M}}_{j} \mathbf{J}_{j}\left(\mathbf{J}_{j}^{\top} \overline{\mathbf{M}}_{j} \mathbf{J}_{j}\right)^{+} \mathbf{J}_{j}^{\top} \overline{\mathbf{M}}_{j}\right) \mathbf{J}_{j} \ddot{\boldsymbol{q}}_{j} \\
& \quad=\overline{\mathbf{M}}_{j}^{1 / 2} \mathbf{C}\left(\mathbf{I}_{n_{j}}-\left(\mathbf{C}^{\top} \mathbf{C}\right)^{+}\left(\mathbf{C}^{\top} \mathbf{C}\right)\right) \ddot{\boldsymbol{q}}_{j}=\overline{\mathbf{M}}_{j}^{1 / 2} \mathbf{0}_{d \times n_{j}} \ddot{\boldsymbol{q}}_{j}=\mathbf{0}
\end{aligned}
$$

with $\mathbf{C}:=\overline{\mathbf{M}}_{j}^{1 / 2} \mathbf{J}_{j}$, see Lemma 2a in Appendix A.

Multiplying (11) from the left by $\mathbf{K}_{i}^{-\top}$ and inserting $\boldsymbol{\mu}_{j}$ from (16) for all $j \in I_{i}$, the equilibrium conditions for body $(\bullet)^{(i)}$ get the more compact form (13) with

$$
\begin{aligned}
\overline{\mathbf{M}}_{i}:= & \mathbf{K}_{i}^{-\top} \mathbf{M}_{i} \mathbf{K}_{i}^{-1} \\
& +\sum_{j \in I_{i}} \mathbf{K}_{i}^{-\top} \mathbf{H}_{j}^{\top}\left(\overline{\mathbf{M}}_{j}-\overline{\mathbf{M}}_{j} \mathbf{J}_{j}\left(\mathbf{J}_{j}^{\top} \overline{\mathbf{M}}_{j} \mathbf{J}_{j}\right)^{+} \mathbf{J}_{j}^{\top} \overline{\mathbf{M}}_{j}\right) \mathbf{H}_{j} \mathbf{K}_{i}^{-1}, \\
\overline{\boldsymbol{f}}_{i}:= & \mathbf{K}_{i}^{-\top} \boldsymbol{f}_{i} \\
& -\sum_{j \in I_{i}} \mathbf{K}_{i}^{-\top} \mathbf{H}_{j}^{\top}\left(\mathbf{I}_{d}-\overline{\mathbf{M}}_{j} \mathbf{J}_{j}\left(\mathbf{J}_{j}^{\top} \overline{\mathbf{M}}_{j} \mathbf{J}_{j}\right)^{+} \mathbf{J}_{j}^{\top}\right)\left(\overline{\boldsymbol{f}}_{j}+\overline{\mathbf{M}}_{j} \boldsymbol{k}_{j}^{(\mathrm{II})}\right) .
\end{aligned}
$$

Lemma 1 in Appendix A shows that matrix $\overline{\mathbf{M}}_{i}$ in (17a) is symmetric, positive semi-definite since it is a finite sum of symmetric, positive semi-definite matrices. Starting from the leaf bodies and following all branches of the kinematic tree to the root, the compact form (13) of the equilibrium conditions may be obtained recursively for all $N$ bodies $(\bullet)^{(i)}$ of the multibody system. The condensed mass matrices $\overline{\mathbf{M}}_{i} \in \mathbb{R}^{d \times d}$. are symmetric, positive semi-definite.

The backward recursion with $\overline{\mathbf{M}}_{i}, \overline{\boldsymbol{f}}_{i}$ being defined by (17) is well-defined whenever all body mass matrices $\mathbf{M}_{i}$, $(i=1, \ldots, N)$, are symmetric, positive semi-definite and matrices $\mathbf{K}_{i}$ are non-singular. Assuming additionally that the body mass matrices $\mathbf{M}_{i} \in \mathbb{R}^{d \times d}$ are positive definite, matrices $\overline{\mathbf{M}}_{i} \in \mathbb{R}^{d \times d}$ and $\mathbf{J}_{j}^{\top} \overline{\mathbf{M}}_{j} \mathbf{J}_{j} \in \mathbb{R}^{n_{i} \times n_{i}}$ in (17) are non-singular and $\left(\mathbf{J}_{j}^{\top} \overline{\mathbf{M}}_{j} \mathbf{J}_{j}\right)^{+}$may be substituted by $\left(\mathbf{J}_{j}^{\top} \overline{\mathbf{M}}_{j} \mathbf{J}_{j}\right)^{-1}$. In that special case, the recursive definitions (17) are well known from classical multibody formalisms, see, e.g., Lubich et al. (1992).

\subsection{Forward recursion: Absolute coordinates}

The second time derivative (9) of the kinematic relations (7) defines the acceleration $\ddot{\boldsymbol{p}}_{i}$ of body $(\bullet)^{(i)}$ in terms of the acceleration $\ddot{\boldsymbol{p}}_{\pi_{i}}$ of its predecessor $(\bullet)^{\left(\pi_{i}\right)}$ and in terms of the corresponding joint coordinates $\ddot{\boldsymbol{q}}_{i}$. Eliminating the joint coordinates, all (absolute) accelerations $\ddot{\boldsymbol{p}}_{i},(i=1, \ldots, N)$, may be computed recursively starting at the root body since $\ddot{\boldsymbol{p}}_{0} \equiv \mathbf{0}$ (forward recursion).

Left multiplication of (9) by $\left(\mathbf{J}_{i}^{\top} \overline{\mathbf{M}}_{i} \mathbf{J}_{i}\right)^{+} \mathbf{J}_{i}^{\top} \overline{\mathbf{M}}_{i}$ results in

$$
\begin{aligned}
& \left(\mathbf{J}_{i}^{\top} \overline{\mathbf{M}}_{i} \mathbf{J}_{i}\right)^{+}\left(\mathbf{J}_{i}^{\top} \overline{\mathbf{M}}_{i} \mathbf{J}_{i}\right) \ddot{\boldsymbol{q}}_{i} \\
& \quad=-\left(\mathbf{J}_{i}^{\top} \overline{\mathbf{M}}_{i} \mathbf{J}_{i}\right)^{+} \mathbf{J}_{i}^{\top} \overline{\mathbf{M}}_{i}\left(\mathbf{H}_{i} \ddot{\boldsymbol{p}}_{\pi_{i}}+\boldsymbol{k}_{i}^{(\mathrm{II})}\right)-\left(\mathbf{J}_{i}^{\top} \overline{\mathbf{M}}_{i} \mathbf{J}_{i}\right)^{+} \mathbf{J}_{i}^{\top} \overline{\boldsymbol{f}}_{i}
\end{aligned}
$$

since

$\mathbf{J}_{i}^{\top} \overline{\mathbf{M}}_{i} \mathbf{K}_{i} \ddot{\boldsymbol{p}}_{i}=\mathbf{J}_{i}^{\top} \overline{\boldsymbol{f}}_{i}-\mathbf{J}_{i}^{\top} \boldsymbol{\mu}_{i}=\mathbf{J}_{i}^{\top} \overline{\boldsymbol{f}}_{i}$,

see (12) and (13). If $\overline{\mathbf{M}}_{i} \in \mathbb{R}^{d \times d}$ is symmetric, positive definite then $\mathbf{J}_{i}^{\top} \overline{\mathbf{M}}_{i} \mathbf{J}_{i} \in \mathbb{R}^{n_{i} \times n_{i}}$ is symmetric, positive definite as well and (18) defines an explicit expression for $\ddot{\boldsymbol{q}}_{i}$ because of $\left(\mathbf{J}_{i}^{\top} \overline{\mathbf{M}}_{i} \mathbf{J}_{i}\right)^{+}\left(\mathbf{J}_{i}^{\top} \overline{\mathbf{M}}_{i} \mathbf{J}_{i}\right)=\mathbf{I}_{n_{i}}$ in that case. In general, however, Eq. (18) determines only $r_{i}:=\operatorname{rank}\left(\mathbf{J}_{i}^{\top} \overline{\mathbf{M}}_{i} \mathbf{J}_{i}\right) \leq n_{i}$ components of $\ddot{\boldsymbol{q}}_{i} \in \mathbb{R}^{n_{i}}$, see also Remark $1 \mathrm{~b}$ in Appendix A.

Substituting (18) in (9), we get the expression

$$
\begin{aligned}
\ddot{\boldsymbol{p}}_{i}=- & -\overline{\mathbf{H}}_{i} \ddot{\boldsymbol{p}}_{\pi_{i}}-\overline{\boldsymbol{k}}_{i}^{(\mathrm{II})} \\
& -\mathbf{K}_{i}^{-1} \mathbf{J}_{i}\left(\mathbf{I}_{n_{i}}-\left(\mathbf{J}_{i}^{\top} \overline{\mathbf{M}}_{i} \mathbf{J}_{i}\right)^{+}\left(\mathbf{J}_{i}^{\top} \overline{\mathbf{M}}_{i} \mathbf{J}_{i}\right)\right) \ddot{\boldsymbol{q}}_{i}
\end{aligned}
$$

with

$$
\begin{array}{rrr}
\overline{\mathbf{H}}_{i}:= & \mathbf{K}_{i}^{-1}\left(\mathbf{I}_{d}-\mathbf{J}_{i}\left(\mathbf{J}_{i}^{\top} \overline{\mathbf{M}}_{i} \mathbf{J}_{i}\right)^{+} \mathbf{J}_{i}^{\top} \overline{\mathbf{M}}_{i}\right) \mathbf{H}_{i}, \\
\overline{\boldsymbol{k}}_{i}^{(\mathrm{II})}:= & \mathbf{K}_{i}^{-1}\left(\mathbf{I}_{d}-\mathbf{J}_{i}\left(\mathbf{J}_{i}^{\top} \overline{\mathbf{M}}_{i} \mathbf{J}_{i}\right)^{+} \mathbf{J}_{i}^{\top} \overline{\mathbf{M}}_{i}\right) \boldsymbol{k}_{i}^{(\mathrm{II})} \\
& -\mathbf{K}_{i}^{-1} \mathbf{J}_{i}\left(\mathbf{J}_{i}^{\top} \overline{\mathbf{M}}_{i} \mathbf{J}_{i}\right)^{+} \mathbf{J}_{i}^{\top} \overline{\boldsymbol{f}}_{i}
\end{array}
$$

that proves to be useful in the forward recursion if $\mathbf{J}_{i}^{\top} \overline{\mathbf{M}}_{i} \mathbf{J}_{i}$ is rank-deficient. The main difference between the full rank and the rank-deficient case is the additional non-zero term $\left(\mathbf{I}_{n_{i}}-\left(\mathbf{J}_{i}^{\top} \overline{\mathbf{M}}_{i} \mathbf{J}_{i}\right)^{+}\left(\mathbf{J}_{i}^{\top} \overline{\mathbf{M}}_{i} \mathbf{J}_{i}\right)\right) \ddot{\boldsymbol{q}}_{i}$ in the right hand side of (19) if $r_{i}=\operatorname{rank}\left(\mathbf{J}_{i}^{\top} \overline{\mathbf{M}}_{i} \mathbf{J}_{i}\right)<n_{i}$. 
It is an important (and non-trivial) observation that this additional term does not affect the successors of body $(\bullet)^{(i)}$ in the kinematic tree (if there are any). Suppose $I_{i} \neq \emptyset$ and consider a (direct) successor $(\bullet)^{(j)}$ of body $(\bullet)^{(i)}, \pi_{j}=i$. In the condensed equilibrium conditions (14), the reaction force between bodies $(\bullet)^{(j)}$ and $(\bullet)^{(i)}$ is represented by $\boldsymbol{\mu}_{j} \in \mathbb{R}^{d}$ that depends on $\ddot{\boldsymbol{p}}_{i}, \overline{\boldsymbol{f}}_{j}$ and $\boldsymbol{k}_{j}^{(\mathrm{II})}$, see (16):

$\boldsymbol{\mu}_{j}=\overline{\boldsymbol{f}}_{j}+\overline{\mathbf{M}}_{j} \mathbf{K}_{j}\left(\overline{\mathbf{H}}_{j} \ddot{\boldsymbol{p}}_{i}+\overline{\boldsymbol{k}}_{j}^{(\mathrm{II})}\right)$.

From (19), we see that the right hand side of (21a) contains the product of matrices

$\overline{\mathbf{J}}_{j}:=\overline{\mathbf{M}}_{j} \mathbf{K}_{j} \overline{\mathbf{H}}_{j} \mathbf{K}_{i}^{-1} \mathbf{J}_{i}\left(\mathbf{I}_{n_{i}}-\left(\mathbf{J}_{i}^{\top} \overline{\mathbf{M}}_{i} \mathbf{J}_{i}\right)^{+}\left(\mathbf{J}_{i}^{\top} \overline{\mathbf{M}}_{i} \mathbf{J}_{i}\right)\right)$

that is rewritten as

$$
\begin{aligned}
\overline{\mathbf{J}}_{j}=\left(\overline{\mathbf{M}}_{j}-\overline{\mathbf{M}}_{j} \mathbf{J}_{j}\left(\mathbf{J}_{j}^{\top} \overline{\mathbf{M}}_{j} \mathbf{J}_{j}\right)^{+} \mathbf{J}_{j}^{\top} \overline{\mathbf{M}}_{j}\right)^{1 / 2} \cdot & \\
\cdot & \mathbf{B}_{j} \mathbf{J}_{i}\left(\mathbf{I}_{n_{i}}-\left(\mathbf{C}^{\top} \mathbf{C}\right)^{+}\left(\mathbf{C}^{\top} \mathbf{C}\right)\right)
\end{aligned}
$$

with

$$
\begin{aligned}
\mathbf{B}_{l} & :=\left(\overline{\mathbf{M}}_{l}-\overline{\mathbf{M}}_{l} \mathbf{J}_{l}\left(\mathbf{J}_{l}^{\top} \overline{\mathbf{M}}_{l} \mathbf{J}_{l}\right)^{+} \mathbf{J}_{l}^{\top} \overline{\mathbf{M}}_{l}\right)^{1 / 2} \mathbf{H}_{l} \mathbf{K}_{i}^{-1},\left(l \in I_{i}\right), \\
\mathbf{B}_{0} & :=\mathbf{M}_{i}^{1 / 2} \mathbf{K}_{i}^{-1}, \\
\mathbf{C} & :=\left(\mathbf{B}_{0}^{\top} \mathbf{B}_{0}+\sum_{l \in I_{i}} \mathbf{B}_{l}^{\top} \mathbf{B}_{l}\right)^{1 / 2} \mathbf{J}_{i}=\overline{\mathbf{M}}_{i}^{1 / 2} \mathbf{J}_{i},
\end{aligned}
$$

see (17a). Lemma 3 from Appendix A proves $\overline{\mathbf{J}}_{j}=\mathbf{0}_{d \times n_{i}}$ resulting in

$\boldsymbol{\mu}_{j}=\overline{\boldsymbol{f}}_{j}+\overline{\mathbf{M}}_{j} \mathbf{K}_{j}\left(-\overline{\mathbf{H}}_{j} \overline{\mathbf{H}}_{i} \ddot{\boldsymbol{p}}_{\pi_{i}}-\overline{\mathbf{H}}_{j} \overline{\boldsymbol{k}}_{i}^{(\mathrm{II})}+\overline{\boldsymbol{k}}_{j}^{(\mathrm{II})}\right)$,

see (21a) and (19).

The two alternative representations of $\boldsymbol{\mu}_{j}$ in $(21 \mathrm{a}, \mathrm{b})$ provide two different ways to evaluate $\ddot{\boldsymbol{p}}_{j}$ by forward recursion, see (14):

$$
\begin{aligned}
& \mathbf{0}=\overline{\mathbf{M}}_{j} \mathbf{K}_{j}\left(\ddot{\boldsymbol{p}}_{j}+\overline{\mathbf{H}}_{j} \ddot{\boldsymbol{p}}_{i}+\overline{\boldsymbol{k}}_{j}^{(\mathrm{II})}\right), \\
& \mathbf{0}=\overline{\mathbf{M}}_{j} \mathbf{K}_{j}\left(\ddot{\boldsymbol{p}}_{j}-\overline{\mathbf{H}}_{j} \overline{\mathbf{H}}_{i} \ddot{\boldsymbol{p}}_{\pi_{i}}-\overline{\mathbf{H}}_{j} \overline{\boldsymbol{k}}_{i}^{(\mathrm{II})}+\overline{\boldsymbol{k}}_{j}^{(\mathrm{II})}\right) .
\end{aligned}
$$

To keep the presentation compact, we assume in the following that the bodies with rank-deficient body mass matrix $\mathbf{M}_{i}$ are isolated in the kinematic tree, i.e., the predecessor of a body $(\bullet)^{(i)}$ with rank-deficient $\mathbf{M}_{i}$ is either the root body $\left(\pi_{i}=0\right)$ or a body with non-singular body mass matrix:

$\operatorname{rank} \mathbf{M}_{i}<d \Rightarrow\left(\pi_{i}=0 \quad\right.$ or $\left.\operatorname{rank} \mathbf{M}_{\pi_{i}}=d\right)$.

For $\pi_{i} \neq 0$, this assumption implies that $\overline{\mathbf{M}}_{\pi_{i}}$ is non-singular as well since a symmetric, positive semi-definite matrix $\mathbf{M}_{\pi_{i}}$ with rank $\mathbf{M}_{\pi_{i}}=d$ is positive definite and $\overline{\mathbf{M}}_{\pi_{i}}$ is defined by a sum of $\mathbf{K}_{\pi_{i}}^{-\top} \mathbf{M}_{\pi_{i}} \mathbf{K}_{\pi_{i}}^{-1}$ and a finite number of symmetric, positive semi-definite matrices, see (17a).

The technical assumption (23) allows to evaluate recursively $\ddot{\boldsymbol{p}}_{j}$ for all bodies with non-singular $\overline{\mathbf{M}}_{j}$ starting at the root body $(\bullet)^{(0)}$ that was supposed to be inertially fixed $\left(\ddot{\boldsymbol{p}}_{0}=\mathbf{0}\right)$. Let a body $(\bullet)^{(j)}$ be given with $\operatorname{rank} \overline{\mathbf{M}}_{j}=d$ and denote its predecessor by $i:=\pi_{j}$. If $\pi_{j}=0$ or $i=\pi_{j} \neq 0$ and the condensed mass matrix $\overline{\mathbf{M}}_{i}$ of the predecessor is non-singular then $\ddot{\boldsymbol{p}}_{i}$ has been computed before and $\ddot{\boldsymbol{p}}_{j}$ may be obtained from (22a) since $\mathbf{K}_{j}$ was supposed to be non-singular:

$\ddot{\boldsymbol{p}}_{j}=-\overline{\mathbf{H}}_{j} \ddot{\boldsymbol{p}}_{i}-\overline{\boldsymbol{k}}_{j}^{(\mathrm{II})}$.

Otherwise, $i=\pi_{j} \neq 0$ and $\overline{\mathbf{M}}_{i}$ is rank-deficient and the corresponding body mass matrix $\mathbf{M}_{i}$ has to be rank-deficient as well. Then, body $(\bullet)^{(i)}$ may be skipped in the forward recursion since assumption (23) guarantees that $\ddot{\boldsymbol{p}}_{\pi_{i}}$ has been computed before and $\ddot{\boldsymbol{p}}_{j}$ may be obtained from (22b):

$\ddot{\boldsymbol{p}}_{j}=\overline{\mathbf{H}}_{j} \overline{\mathbf{H}}_{i} \ddot{\boldsymbol{p}}_{\pi_{i}}+\overline{\mathbf{H}}_{j} \overline{\boldsymbol{k}}_{i}^{(\mathrm{III})}-\overline{\boldsymbol{k}}_{j}^{(\mathrm{II})}$.

The proposed forward recursion algorithm evaluates $\ddot{\boldsymbol{p}}_{j}$ for all bodies $(\bullet)^{(j)}$ with non-singular $\overline{\mathbf{M}}_{j}$ provided that the technical assumption (23) is satisfied and the body mass matrices $\mathbf{M}_{i}$ are symmetric, positive semi-definite for all $N$ bodies of the multibody system model, $(i=1, \ldots, N)$. It provides furthermore the algorithmic basis to evaluate the equations of motion as mixed second and first order system for the joint coordinates $\boldsymbol{q}=\left(\boldsymbol{q}_{1}, \ldots, \boldsymbol{q}_{N}\right)$.

\subsection{Equations of motion: mixed second and first order system of differential equations}

Let us consider again a body $(\bullet)^{(j)}$ with non-singular $\overline{\mathbf{M}}_{j}$ and denote $i=\pi_{j}$. Multiplying (15) from the left by $\mathbf{J}_{j}^{\top} \overline{\mathbf{M}}_{j}$, we get

$\left(\mathbf{J}_{j}^{\top} \overline{\mathbf{M}}_{j} \mathbf{J}_{j}\right) \ddot{\boldsymbol{q}}_{j}=-\mathbf{J}_{j}^{\top} \overline{\mathbf{M}}_{j}\left(\mathbf{H}_{j} \ddot{p}_{i}+\boldsymbol{k}_{j}^{(\mathrm{II})}\right)-\mathbf{J}_{j}^{\top} \overline{\boldsymbol{f}}_{j}$

since $\mathbf{J}_{j}^{\top} \overline{\mathbf{M}}_{j} \mathbf{K}_{j} \ddot{\boldsymbol{p}}_{j}=\mathbf{J}_{j}^{\top} \overline{\boldsymbol{f}}_{j}-\mathbf{J}_{j}^{\top} \boldsymbol{\mu}_{j}=\mathbf{J}_{j}^{\top} \overline{\boldsymbol{f}}_{j}$, see (14) and (12).

As before, we get an alternative expression from substituting in the right hand side of (25a) the term $\ddot{\boldsymbol{p}}_{i}$ according to (19):

$$
\begin{gathered}
\left(\mathbf{J}_{j}^{\top} \overline{\mathbf{M}}_{j} \mathbf{J}_{j}\right) \ddot{\boldsymbol{q}}_{j}-\mathbf{J}_{j}^{\top} \overline{\mathbf{M}}_{j} \mathbf{H}_{j} \mathbf{K}_{i}^{-1} \mathbf{J}_{i}\left(\mathbf{I}_{n_{i}}-\left(\mathbf{J}_{i}^{\top} \overline{\mathbf{M}}_{i} \mathbf{J}_{i}\right)^{+}\left(\mathbf{J}_{i}^{\top} \overline{\mathbf{M}}_{i} \mathbf{J}_{i}\right)\right) \ddot{\boldsymbol{q}}_{i} \\
=\mathbf{J}_{j}^{\top} \overline{\mathbf{M}}_{j}\left(\mathbf{H}_{j} \overline{\mathbf{H}}_{i} \ddot{\boldsymbol{p}}_{\pi_{i}}+\mathbf{H}_{j} \overline{\boldsymbol{k}}_{i}^{(\mathrm{II})}-\boldsymbol{k}_{j}^{(\mathrm{II})}\right)-\mathbf{J}_{j}^{\top} \overline{\boldsymbol{f}}_{j} .
\end{gathered}
$$

The simpler expression (25a) can be used whenever $\pi_{j}=0$ or $i=\pi_{j} \neq 0$ and $\overline{\mathbf{M}}_{i}$ is non-singular since $\ddot{\boldsymbol{p}}_{i}$ has been evaluated by forward recursion in that case. Eq. (25b) shows that the situation is substantially more complicated if $i=\pi_{j} \neq 0$ and $\overline{\mathbf{M}}_{i}$ is rank-deficient since in that case body $(\bullet)^{(i)}$ and the corresponding acceleration term $\ddot{\boldsymbol{p}}_{i}$ have been skipped in the forward recursion and $\ddot{\boldsymbol{p}}_{\pi_{i}}$ has to be used instead.

In the latter case, the technical assumption (23) guarantees that $\ddot{\boldsymbol{p}}_{\pi_{i}}$ is really available from the forward recursion since either $\pi_{i}=0$ or $\pi_{i} \neq 0$ and $\overline{\mathbf{M}}_{\pi_{i}}$ is non-singular. Therefore, the right hand side of $(25 \mathrm{a})$ with $(j, i)$ being substituted by $\left(i, \pi_{i}\right)$ may be evaluated straightforwardly:

$\left(\mathbf{J}_{i}^{\top} \overline{\mathbf{M}}_{i} \mathbf{J}_{i}\right) \ddot{\boldsymbol{q}}_{i}=-\mathbf{J}_{i}^{\top} \overline{\mathbf{M}}_{i}\left(\mathbf{H}_{i} \ddot{\boldsymbol{p}}_{\pi_{i}}+\boldsymbol{k}_{i}^{(\mathrm{II})}\right)-\mathbf{J}_{i}^{\top} \overline{\boldsymbol{f}}_{i}$. 
In (26), the coefficient $\mathbf{J}_{i}^{\top} \overline{\mathbf{M}}_{i} \mathbf{J}_{i}$ of $\ddot{\boldsymbol{q}}_{i}$ is symmetric, positive semi-definite and may therefore be diagonalized, see Remark 1 in Appendix A:

$\mathbf{J}_{i}^{\top} \overline{\mathbf{M}}_{i} \mathbf{J}_{i}=: \mathbf{A}_{i}=\mathbf{X}_{i} \boldsymbol{\Lambda}_{i} \mathbf{X}_{i}^{\top}=\mathbf{X}_{i}\left(\begin{array}{cc}\overline{\boldsymbol{\Lambda}}_{i} & \mathbf{0} \\ \mathbf{0} & \mathbf{0}\end{array}\right) \mathbf{X}_{i}^{\top} \in \mathbb{R}^{n_{i} \times n_{i}}$.

Here, $\overline{\mathbf{\Lambda}}_{i} \in \mathbb{R}^{r_{i} \times r_{i}}$ with $r_{i}=\operatorname{rank}\left(\mathbf{J}_{i}^{\top} \overline{\mathbf{M}}_{i} \mathbf{J}_{i}\right) \leq n_{i}$ is a positive diagonal matrix containing the non-zero eigenvalues of $\mathbf{J}_{i}^{\top} \overline{\mathbf{M}}_{i} \mathbf{J}_{i}$. Matrix $\mathbf{X}_{i} \in \mathbb{R}^{n_{i} \times n_{i}}$ is orthogonal. The projector $\mathbf{I}_{n_{i}}-\left(\mathbf{J}_{i}^{\top} \overline{\mathbf{M}}_{i} \mathbf{J}_{i}\right)^{+}\left(\mathbf{J}_{i}^{\top} \overline{\mathbf{M}}_{i} \mathbf{J}_{i}\right)$ in (25b) may be expressed as

$$
\begin{gathered}
\mathbf{I}_{n_{i}}-\left(\mathbf{J}_{i}^{\top} \overline{\mathbf{M}}_{i} \mathbf{J}_{i}\right)^{+}\left(\mathbf{J}_{i}^{\top} \overline{\mathbf{M}}_{i} \mathbf{J}_{i}\right)=\mathbf{X}_{i}(\mathbf{I}_{n_{i}}-\boldsymbol{\Lambda}_{i}^{+} \underbrace{\mathbf{X}_{i}^{\top} \mathbf{X}_{i}}_{=\mathbf{I}_{n_{i}}} \mathbf{\Lambda}_{i}) \mathbf{X}_{i}^{\top} \\
=\mathbf{X}_{i}\left(\mathbf{I}_{n_{i}}-\left(\begin{array}{cc}
\mathbf{I}_{r_{i}} & \mathbf{0} \\
\mathbf{0} & \mathbf{0}
\end{array}\right)\right) \mathbf{X}_{i}^{\top}=\mathbf{X}_{i}\left(\begin{array}{cc}
\mathbf{0} & \mathbf{0} \\
\mathbf{0} & \mathbf{I}_{n_{i}-r_{i}}
\end{array}\right) \mathbf{X}_{i}^{\top} .
\end{gathered}
$$

Multiplying (26) from the left by $\mathbf{X}_{i}^{\top}$, we end up with a decoupled system of $r_{i}$ linearly implicit second order differential equations

$$
\begin{aligned}
& \overline{\mathbf{\Lambda}}_{i}\left(\mathbf{I}_{r_{i}} \mathbf{0}_{r_{i} \times\left(n_{i}-r_{i}\right)}\right) \mathbf{X}_{i}^{\top} \ddot{\boldsymbol{q}}_{i} \\
& \quad=\quad-\left(\mathbf{I}_{r_{i}} \mathbf{0}_{r_{i} \times\left(n_{i}-r_{i}\right)}\right) \mathbf{X}_{i}^{\top} \mathbf{J}_{i}^{\top}\left(\overline{\mathbf{M}}_{i}\left(\mathbf{H}_{i} \ddot{p}_{\pi_{i}}+\boldsymbol{k}_{i}^{(\mathrm{II})}\right)+\overline{\boldsymbol{f}}_{i}\right)
\end{aligned}
$$

and $n_{i}-r_{i}$ additional equations that do not contain $\ddot{\boldsymbol{q}}_{i}$ and may further be simplified to

$\mathbf{0}_{n_{i}-r_{i}}=\left(\mathbf{0}_{\left(n_{i}-r_{i}\right) \times r_{i}} \mathbf{I}_{n_{i}-r_{i}}\right) \mathbf{X}_{i}^{\top} \mathbf{J}_{i}^{\top} \overline{\boldsymbol{f}}_{i}$

since $\left(\mathbf{0}_{\left(n_{i}-r_{i}\right) \times r_{i}} \mathbf{I}_{n_{i}-r_{i}}\right) \mathbf{X}_{i}^{\top} \mathbf{J}_{i}^{\top} \overline{\mathbf{M}}_{i}\left(\mathbf{H}_{i} \ddot{\boldsymbol{p}}_{\pi_{i}}+\boldsymbol{k}_{i}^{(\mathrm{II})}\right)$ vanishes because (27) and Lemma 2b with $\mathbf{C}:=\overline{\mathbf{M}}_{i}^{1 / 2} \mathbf{J}_{i} \in \mathbb{R}^{d \times n_{i}}$ imply

$$
\begin{aligned}
\left(\mathbf{0}_{\left(n_{i}-r_{i}\right) \times r_{i}}\right. & \left.\mathbf{I}_{n_{i}-r_{i}}\right) \mathbf{X}_{i}^{\top} \mathbf{J}_{i}^{\top} \overline{\mathbf{M}}_{i}= \\
= & \left(\mathbf{0}_{\left(n_{i}-r_{i}\right) \times r_{i}} \mathbf{I}_{n_{i}-r_{i}}\right) \underbrace{\mathbf{X}_{i}^{\top} \mathbf{X}_{i}}_{=\mathbf{I}_{n_{i}}}\left(\begin{array}{cc}
\mathbf{0} & \mathbf{0} \\
\mathbf{0} & \mathbf{I}_{n_{i}-r_{i}}
\end{array}\right) \mathbf{X}_{i}^{\top} \mathbf{J}_{i}^{\top} \overline{\mathbf{M}}_{i} \\
= & \left(\mathbf{0}_{\left(n_{i}-r_{i}\right) \times r_{i}} \mathbf{I}_{n_{i}-r_{i}}\right) \mathbf{X}_{i}^{\top}\left(\mathbf{I}_{n_{i}}-\left(\mathbf{J}_{i}^{\top} \overline{\mathbf{M}}_{i} \mathbf{J}_{i}\right)\left(\mathbf{J}_{i}^{\top} \overline{\mathbf{M}}_{i} \mathbf{J}_{i}\right)^{+}\right) \mathbf{J}_{i}^{\top} \overline{\mathbf{M}}_{i} \\
= & \left(\mathbf{0}_{\left(n_{i}-r_{i}\right) \times r_{i}} \mathbf{I}_{n_{i}-r_{i}}\right) \mathbf{X}_{i}^{\top}\left(\mathbf{I}_{n_{i}}-\left(\mathbf{C}^{\top} \mathbf{C}\right)\left(\mathbf{C}^{\top} \mathbf{C}\right)^{+}\right) \mathbf{C}^{\top} \overline{\mathbf{M}}_{i}^{1 / 2} \\
= & \mathbf{0}_{n_{i} \times d} .
\end{aligned}
$$

The equations of motion for the multibody system model are given by $(25 \mathrm{~b})$ for all bodies $(\bullet)^{(j)}$ with $i:=\pi_{j} \neq 0$ and $\operatorname{rank} \overline{\mathbf{M}}_{i}<d$ and by (25a) for the remaining bodies, $(j=1, \ldots, N)$. They are composed of $\sum_{i} r_{i}$ linearly implicit second order differential equations (25b), (28a) and $\sum_{i}\left(n_{i}-r_{i}\right)$ additional equations (28b) to define $\left(\mathbf{0}_{\left(n_{i}-r_{i}\right) \times r_{i}} \mathbf{I}_{n_{i}-r_{i}}\right) \mathbf{X}_{i}^{\top} \dot{\boldsymbol{q}}_{i},(i=1, \ldots, N)$, see also the detailed discussion in Sect. 3.5 below. Similar to a classical residual formalism, see Eichberger (1994), the residuals in (25) may be used to integrate the equations of motion by general purpose DAE solvers like DAssL, see Brenan et al. (1996).

\subsection{Equations of motion: formal analysis}

For a formal analysis of equations of motion (25), we introduce velocity coordinates $\boldsymbol{v}_{0}:=\dot{\boldsymbol{p}}_{0}$,

$\boldsymbol{v}_{j}:=\left\{\begin{array}{l}\mathbf{X}_{j}^{\top} \dot{\boldsymbol{q}}_{j}-\mathbf{T}_{j} \dot{\boldsymbol{q}}_{i} \text { if } i:=\pi_{j} \neq 0 \text { and } \operatorname{rank} \overline{\mathbf{M}}_{i}<d, \\ \mathbf{X}_{j}^{\top} \dot{\boldsymbol{q}}_{j} \text { otherwise, }\end{array}\right.$

$(j=1, \ldots, N)$, with

$$
\mathbf{T}_{j}:=\boldsymbol{\Lambda}_{j}^{-1} \mathbf{X}_{j}^{\top} \mathbf{J}_{j}^{\top} \overline{\mathbf{M}}_{j} \mathbf{H}_{j} \mathbf{K}_{i}^{-1} \mathbf{J}_{i}\left(\mathbf{I}_{n_{i}}-\left(\mathbf{J}_{i}^{\top} \overline{\mathbf{M}}_{i} \mathbf{J}_{i}\right)^{+}\left(\mathbf{J}_{i}^{\top} \overline{\mathbf{M}}_{i} \mathbf{J}_{i}\right)\right) .
$$

Because of (29), the joint coordinates $\dot{\boldsymbol{q}}_{j}$ may also be expressed in terms of $\boldsymbol{v}:=\left(\boldsymbol{v}_{1}, \ldots, \boldsymbol{v}_{N}\right)$ :

$\dot{\boldsymbol{q}}_{j}=\boldsymbol{\varphi}_{j}^{[1]}(\boldsymbol{q}, \boldsymbol{v}, t),(j=1, \ldots, N)$,

with

$\varphi_{j}^{[1]}=\left\{\begin{array}{l}\mathbf{X}_{j}\left(\boldsymbol{v}_{j}+\mathbf{T}_{j} \mathbf{X}_{i} \boldsymbol{v}_{i}\right) \text { if } i:=\pi_{j} \neq 0 \text { and } \operatorname{rank} \overline{\mathbf{M}}_{i}<d, \\ \mathbf{X}_{j} \boldsymbol{v}_{j} \text { otherwise }\end{array}\right.$

since $\operatorname{rank} \overline{\mathbf{M}}_{i}<d$ and the technical assumption (23) imply $\dot{\boldsymbol{q}}_{i}=\mathbf{X}_{i} \boldsymbol{v}_{i}$. For all bodies $(\bullet)^{(i)}$ with $\operatorname{rank} \overline{\mathbf{M}}_{i}<d$, vector $\boldsymbol{v}_{i}$ is split according to

$\boldsymbol{v}_{i}=\mathbf{X}_{i}^{\top} \dot{\boldsymbol{q}}_{i}=\left(\begin{array}{c}\boldsymbol{\eta}_{i} \\ \zeta_{i}\end{array}\right)$

with

$\begin{aligned} \boldsymbol{\eta}_{i} & :=\left(\mathbf{I}_{r_{i}} \mathbf{0}_{r_{i} \times\left(n_{i}-r_{i}\right)}\right) \mathbf{X}_{i}^{\top} \dot{\boldsymbol{q}}_{i} \in \mathbb{R}^{r_{i}}, \\ \boldsymbol{\zeta}_{i} & :=\left(\mathbf{0}_{\left(n_{i}-r_{i}\right) \times r_{i}} \mathbf{I}_{n_{i}-r_{i}}\right) \mathbf{X}_{i}^{\top} \dot{\boldsymbol{q}}_{i} \in \mathbb{R}^{n_{i}-r_{i}} .\end{aligned}$

In the full rank case $\left(\operatorname{rank} \overline{\mathbf{M}}_{i}=d\right)$, we set $\boldsymbol{\eta}_{i}:=\boldsymbol{v}_{i} \in \mathbb{R}^{n_{i}}$ and leave $\zeta_{i}$ "empty" since $r_{i}=\operatorname{rank}\left(\mathbf{J}_{i}^{\top} \overline{\mathbf{M}}_{i} \mathbf{J}_{i}\right)=n_{i}$, i.e., $n_{i}-r_{i}=0$. In the rank-deficient case ( $\left.\operatorname{rank} \overline{\mathbf{M}}_{i}<d \Rightarrow r_{i}<n_{i}\right)$, the technical assumption (23) guarantees that $\overline{\mathbf{M}}_{j}$ is nonsingular resulting in $\boldsymbol{\eta}_{j}=\boldsymbol{v}_{j}$. With (29) and the diagonalized projector in (27), we see that $\dot{\boldsymbol{q}}_{j}$ may be written as a linear combination of $\boldsymbol{\eta}_{j}$ and $\boldsymbol{\zeta}_{i}$ that is independent of $\boldsymbol{\eta}_{i}$ :

$\dot{\boldsymbol{q}}_{j}=\mathbf{X}_{j}\left(\boldsymbol{v}_{j}+\mathbf{T}_{j} \dot{\boldsymbol{q}}_{i}\right)=\mathbf{X}_{j} \boldsymbol{\eta}_{j}+\mathbf{X}_{j} \Lambda_{j}^{-1} \mathbf{X}_{j}^{\top} \mathbf{J}_{j}^{\top} \overline{\mathbf{M}}_{j} \mathbf{H}_{j} \mathbf{K}_{i}^{-1} \mathbf{J}_{i} \mathbf{X}_{i}\left(\begin{array}{c}\mathbf{o}_{r_{i}} \\ \boldsymbol{\zeta}_{i}\end{array}\right)$.

The time derivative of $\boldsymbol{v}_{j}$ in (29) depends on time derivatives of

$\mathbf{X}_{j}^{\top}=\mathbf{X}_{j}^{\top}\left(\boldsymbol{p}_{0}(t), \boldsymbol{p}(t), \boldsymbol{q}(t), t\right), \quad \mathbf{T}_{j}=\mathbf{T}_{j}\left(\boldsymbol{p}_{0}(t), \boldsymbol{p}(t), \boldsymbol{q}(t), t\right)$

with $\boldsymbol{p}=\boldsymbol{p}\left(\boldsymbol{p}_{0}, \boldsymbol{q}, t\right)$, see (7). Let $\dot{\mathbf{X}}_{j}^{\top}=\dot{\mathbf{X}}_{j}^{\top}\left(\boldsymbol{p}_{0}, \boldsymbol{v}_{0}, \boldsymbol{q}, \dot{\boldsymbol{q}}, t\right)$ and $\dot{\mathbf{T}}_{j}=\dot{\mathbf{T}}_{j}\left(\boldsymbol{p}_{0}, \boldsymbol{v}_{0}, \boldsymbol{q}, \dot{\boldsymbol{q}}, t\right)$ be defined such that

$\dot{\mathbf{X}}_{j}^{\top} \boldsymbol{w}=\frac{\mathrm{d}}{\mathrm{d} t}\left(\mathbf{X}_{j}^{\top} \boldsymbol{w}\right),\left(\boldsymbol{w} \in \mathbb{R}^{n_{j}}\right), \quad \dot{\mathbf{T}}_{j} \boldsymbol{w}=\frac{\mathrm{d}}{\mathrm{d} t}\left(\mathbf{T}_{j} \boldsymbol{w}\right),\left(\boldsymbol{w} \in \mathbb{R}^{n_{\pi_{j}}}\right)$. 
For bodies $(\bullet)^{(j)}$ with $i:=\pi_{j} \neq 0$ and $\operatorname{rank} \overline{\mathbf{M}}_{i}<d$, we get from (29), (30) and from the product rule

$$
\begin{aligned}
\dot{\boldsymbol{v}}_{j} & =\mathbf{X}_{j}^{\top} \ddot{\boldsymbol{q}}_{j}-\mathbf{T}_{j} \ddot{\boldsymbol{q}}_{i}+\dot{\mathbf{X}}_{j}^{\top} \dot{\boldsymbol{q}}_{j}-\dot{\mathbf{T}}_{j} \dot{\boldsymbol{q}}_{i} \\
& =\mathbf{X}_{j}^{\top} \ddot{\boldsymbol{q}}_{j}-\mathbf{T}_{j} \ddot{\boldsymbol{q}}_{i}+\dot{\mathbf{X}}_{j}^{\top} \mathbf{X}_{j}\left(\boldsymbol{v}_{j}+\mathbf{T}_{j} \mathbf{X}_{i} \boldsymbol{v}_{i}\right)-\dot{\mathbf{T}}_{j} \mathbf{X}_{i} \boldsymbol{v}_{i} .
\end{aligned}
$$

Multiplying the equations of motion (25b) from the left by $\boldsymbol{\Lambda}_{j}^{-1} \mathbf{X}_{j}^{\top}$, we observe

$$
\boldsymbol{\Lambda}_{j}^{-1} \mathbf{X}_{j}^{\top}\left(\mathbf{J}_{j}^{\top} \overline{\mathbf{M}}_{j} \mathbf{J}_{j}\right)=\boldsymbol{\Lambda}_{j}^{-1} \mathbf{X}_{j}^{\top} \mathbf{X}_{j} \boldsymbol{\Lambda}_{j} \mathbf{X}_{j}^{\top}=\mathbf{X}_{j}^{\top}
$$

and end up with

$$
\begin{aligned}
\dot{\boldsymbol{\eta}}_{j}= & \dot{\boldsymbol{v}}_{j}=\dot{\mathbf{X}}_{j}^{\top} \mathbf{X}_{j}\left(\boldsymbol{v}_{j}+\mathbf{T}_{j} \mathbf{X}_{i} \boldsymbol{v}_{i}\right)-\dot{\mathbf{T}}_{j} \mathbf{X}_{i} \boldsymbol{v}_{i} \\
& +\boldsymbol{\Lambda}_{j}^{-1} \mathbf{X}_{j}^{\top} \mathbf{J}_{j}^{\top}\left(\overline{\mathbf{M}}_{j}\left(\mathbf{H}_{j} \overline{\mathbf{H}}_{i} \ddot{\boldsymbol{p}}_{\pi_{i}}+\mathbf{H}_{j} \overline{\boldsymbol{k}}_{i}^{(\mathrm{II})}-\boldsymbol{k}_{j}^{(\mathrm{II})}\right)-\overline{\boldsymbol{f}}_{j}\right) .
\end{aligned}
$$

Since $\ddot{\boldsymbol{p}}_{\pi_{i}}$ has been evaluated by forward recursion and the joint coordinates $\dot{\boldsymbol{q}}$ are given in terms of $\boldsymbol{q}, \boldsymbol{v}, \boldsymbol{p}_{0}(t), \boldsymbol{v}_{0}(t)$ and $t$, see (30), the system of $n_{j}$ first order differential equations (32) may be written as

$\dot{\boldsymbol{\eta}}_{j}=\boldsymbol{\varphi}_{j}^{[2]}(\boldsymbol{q}, \boldsymbol{v}, t) \quad$ if $\quad i:=\pi_{j} \neq 0$ and $\operatorname{rank} \overline{\mathbf{M}}_{i}<d$.

In the same way, (28a) is seen to imply a system of $r_{i} \leq n_{i}$ first order differential equations

$$
\begin{aligned}
& \dot{\boldsymbol{\eta}}_{i}=\boldsymbol{\varphi}_{i}^{[2]}(\boldsymbol{q}, \boldsymbol{v}, t):= \\
& \quad-\overline{\boldsymbol{\Lambda}}_{i}^{-1}\left(\mathbf{I}_{r_{i}} \mathbf{0}_{r_{i} \times\left(n_{i}-r_{i}\right)}\right) \mathbf{X}_{i}^{\top} \mathbf{J}_{i}^{\top}\left(\overline{\mathbf{M}}_{i}\left(\mathbf{H}_{i} \ddot{\boldsymbol{p}}_{\pi_{i}}+\boldsymbol{k}_{i}^{(\mathrm{II})}\right)+\overline{\boldsymbol{f}}_{i}\right) \\
& \quad+\left(\mathbf{I}_{r_{i}} \mathbf{0}_{r_{i} \times\left(n_{i}-r_{i}\right)}\right) \dot{\mathbf{X}}_{i}^{\top} \boldsymbol{v}_{i} .
\end{aligned}
$$

Finally the $n_{i}-r_{i}$ equations (28b) are written as algebraic equations

$\mathbf{0}_{n_{i}-r_{i}}=\gamma_{i}(\boldsymbol{q}, \boldsymbol{v}, t)$.

With these transformations, the equations of motion are re-formulated as differential-algebraic system (2) with $\sum_{i}\left(n_{i}+r_{i}\right)$ differential variables $\boldsymbol{y}_{0}=\left(\boldsymbol{q}_{1}, \ldots, \boldsymbol{q}_{N}, \boldsymbol{\eta}_{1}, \ldots, \boldsymbol{\eta}_{N}\right)$ satisfying (2a) with right hand sides $\varphi_{i}^{[1]}$ of dimension $n_{i}$ and right hand sides $\varphi_{i}^{[2]}$ of dimension $r_{i},(i=1, \ldots, N)$, and $\sum_{i}\left(n_{i}-r_{i}\right)$ algebraic variables $z_{0}=\zeta:=\left(\zeta_{1}, \ldots, \zeta_{N}\right)$ satisfying (2b) with functions $\gamma_{i},(i=1, \ldots, N)$.

The algebraic equations $(2 b)$ define implicitly the "algebraic" velocity components $z_{0}=\zeta$, if the Jacobian $\partial \gamma / \partial \zeta$ is non-singular along the solution. In practical applications, this regularity assumption will typically be satisfied if $\partial \gamma_{i} / \partial \zeta_{i}$ is non-singular for all bodies $(\bullet)^{(i)}$ with $r_{i}=\operatorname{rank} \overline{\mathbf{M}}_{i}<n_{i}$, $(i=1, \ldots, N)$, which may be achieved by appropriate damping terms in the force vector $\overline{\boldsymbol{f}}_{i}$ that should depend on the velocity coordinates $\zeta_{i}=\left(\mathbf{0}_{\left(n_{i}-r_{i}\right) \times r_{i}} \mathbf{I}_{n_{i}-r_{i}}\right) \mathbf{X}_{i}^{\top} \dot{\boldsymbol{q}}_{i}$, see (28b). A more detailed analysis of the regularity of Jacobian $\partial \gamma / \partial \zeta$ is subject of further research.

\section{Neglecting inertia forces in multibody systems: two examples}

The theoretical analysis of Sect. 3 generalizes the results of Arnold et al. (2010) from chain structured systems to general tree structured systems. In this section, we recall two
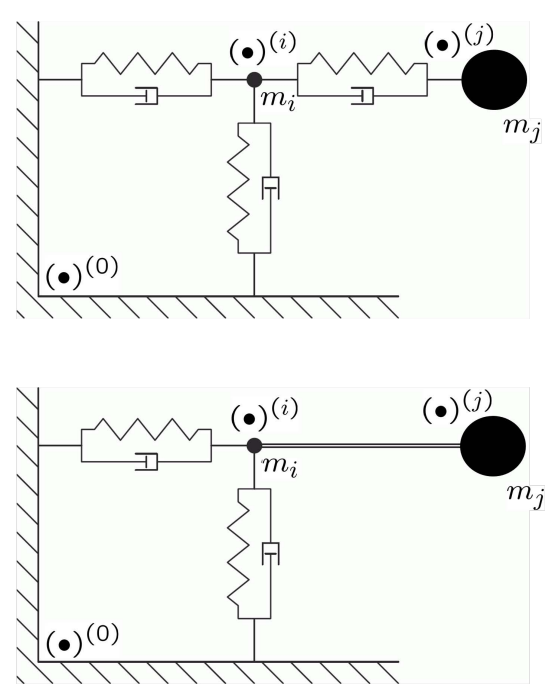

Figure 3. Two planar configurations illustrating the analysis of Sect. 3, see Arnold et al. (2010).

academic test problems from Arnold et al. (2010) to illustrate the basic steps of these investigations. We consider a chain of two mass points $(\bullet)^{(i)},(\bullet)^{(j)}$ in 2-D with $i=\pi_{j}$ and $\pi_{i}=0$. I.e., body $(\bullet)^{(i)}$ follows in the kinematic chain directly the inertial system ("root") and is the predecessor of body $(\bullet)^{(j)}$. In Sect. 3, there are no specific physical assumptions on the joints between bodies $(\bullet)^{(0)}$ and $(\bullet)^{(i)}$ and between bodies $(\bullet)^{(i)}$ and $(\bullet)^{(j)}$, respectively. Therefore, the resulting set of $r_{i}$ explicit second order differential equations (28a) and $n_{i}-r_{i}$ implicit first order differential equations (28b) for body $(\bullet)^{(i)}$ describes arbitrary joint configurations and has a substantially more complex mathematical structure than the corresponding equations of motion in a classical multibody formalism.

In the test problems, bodies $(\bullet)^{(i)}$ and $(\bullet)^{(j)}$ are represented by point masses with $d=2$ degrees of freedom, see Fig. 3. The root body $(\bullet)^{(0)}$ is inertially fixed resulting in $\boldsymbol{p}_{\pi_{i}}(t) \equiv \mathbf{0}$. The absolute coordinates of bodies $(\bullet)^{(i)}$ and $(\bullet)^{(j)}$ are denoted by $\boldsymbol{p}_{i}=\left(p_{i, x}, p_{i, y}\right)^{\top}, \boldsymbol{p}_{j}=\left(p_{j, x}, p_{j, y}\right)^{\top} \in \mathbb{R}^{2}$. In this simplified setting, the diagonal mass matrices $\mathbf{M}_{i}, \mathbf{M}_{j}$ have format $2 \times 2$ and the joint Jacobians satisfy $\mathbf{J}_{i} \in \mathbb{R}^{2 \times n_{i}}$ and $\mathbf{J}_{j} \in \mathbb{R}^{2 \times n_{j}}$.

Bodies $(\bullet)^{(0)}$ and $(\bullet)^{(i)}$ are connected by two linear springdamper elements acting parallel to the $\mathrm{x}$-axis and $\mathrm{y}$-axis, see Fig. 3. The $n_{i}=2$ degrees of freedom in this joint are represented by joint coordinates $\boldsymbol{q}_{i}(t)=\boldsymbol{p}_{i}(t) \in \mathbb{R}^{2}$ such that the functions $\boldsymbol{k}_{i}^{(\mathrm{I})}, \boldsymbol{k}_{i}^{(\mathrm{II})}$ in (8), (9) and (10) vanish identically, $\mathbf{K}_{i}=\mathbf{I}_{2}, \mathbf{H}_{i}=\mathbf{J}_{i}=-\mathbf{I}_{2}$. The free motion of body $(\bullet)^{(j)}$ in y-direction is represented by the joint coordinate $q_{j, y}(t):=p_{j, y}(t)-p_{i, y}(t)$

For the configuration in the upper plot of Fig. 3, bodies $(\bullet)^{(i)}$ and $(\bullet)^{(j)}$ are connected by another linear spring-damper element acting parallel to the $\mathrm{x}$-axis. 
The joint has $n_{j}=2$ degrees of freedom with joint coordinates $\boldsymbol{q}_{j}=\left(q_{j, x}, q_{j, y}\right)^{\top} \in \mathbb{R}^{2}$ and $q_{j, x}(t):=p_{j, x}(t)-p_{i, x}(t)$. Functions $\boldsymbol{k}_{j}^{(\mathrm{I})}, \boldsymbol{k}_{j}^{(\mathrm{II})}$ in (8) and (9) vanish identically, $\mathbf{K}_{j}=\mathbf{I}_{2}$, $\mathbf{H}_{j}=\mathbf{J}_{j}=-\mathbf{I}_{2}$.

In absolute coordinates, the equations of motion are given by

$$
\begin{aligned}
m_{i} \ddot{p}_{i, x} & =-d_{i, x} \dot{p}_{i, x}-c_{i, x} p_{i, x}, \\
& =\quad+d_{j, x}\left(\dot{p}_{j, x}-\dot{p}_{i, x}\right)+c_{j, x}\left(p_{j, x}-p_{i, x}\right), \\
m_{i} \ddot{p}_{i, y} & =-d_{i, y} \dot{p}_{i, y}-c_{i, y} p_{i, y}, \\
m_{j} \ddot{p}_{j, x} & =-d_{j, x}\left(\dot{p}_{j, x}-\dot{p}_{i, x}\right)-c_{j, x}\left(p_{j, x}-p_{i, x}\right), \\
m_{j} \ddot{p}_{j, y} & =0
\end{aligned}
$$

because (12) with $\mathbf{J}_{i}=\mathbf{J}_{j}=-\mathbf{I}_{2}$ implies $\boldsymbol{\mu}_{i}=\boldsymbol{\mu}_{j}=\mathbf{0}$.

With $\mathbf{J}_{j}=-\mathbf{I}_{2}$, Eqs. (17a) and (17b) simplify to $\overline{\mathbf{M}}_{i}=\mathbf{M}_{i}$ and $\overline{\boldsymbol{f}}_{i}=\boldsymbol{f}_{i}$. If the mass $m_{i}$ of body $(\bullet)^{(i)}$ vanishes, we get $\overline{\mathbf{M}}_{i}=\mathbf{M}_{i}=m_{i} \mathbf{I}_{2}=\mathbf{0}_{2 \times 2}$ and $r_{i}=\operatorname{rank}\left(\mathbf{J}_{i}^{\top} \overline{\mathbf{M}}_{i} \mathbf{J}_{i}\right)=0, \mathbf{X}_{i}=\mathbf{I}_{2}$. The equations of motion for coordinates $\boldsymbol{q}_{i}(t)$ are composed of $r_{i}=0$ second order differential equations (28a) and the $n_{i}-r_{i}=2$ implicit first order differential equations

$\mathbf{0}=\boldsymbol{f}_{i}=\left(\begin{array}{c}-d_{i, x} \dot{q}_{i, x}-c_{i, x} q_{i, x}+d_{j, x} \dot{q}_{j, x}+c_{j, x} q_{j, x} \\ -d_{i, y} \dot{q}_{i, y}-c_{i, y} q_{i, y}\end{array}\right)$

see (28b). This result is in perfect agreement with $(33 a, b)$ in the limit case $m_{i}=0$.

The joint coordinates $\boldsymbol{q}_{j}=\boldsymbol{p}_{j}-\boldsymbol{q}_{i}$ are not defined explicitly if $\overline{\mathbf{M}}_{i}=\mathbf{0}$. The equations of motion (25b) yield

$m_{j}\left(\ddot{q}_{j, x}+\ddot{q}_{i, x}\right)=-d_{j, x} \dot{q}_{j, x}-c_{j, x} q_{j, x}$,

$m_{j}\left(\ddot{q}_{j, y}+\ddot{q}_{i, y}\right)=0$.

In the lower plot of Fig. 3, the relative motion of body $(\bullet)^{(j)}$ w.r.t. body $(\bullet)^{(i)}$ is restricted in x-direction by the scalar constraint $p_{j, x}(t)=p_{i, x}(t)+l_{i}$. The joint has only $n_{j}=1$ degree of freedom $\boldsymbol{q}_{j}(t)=q_{j, y}(t)$ with a joint Jacobian $\mathbf{J}_{j}=-(0,1)^{\top}$, $\mathbf{K}_{j}=\mathbf{I}_{2}, \mathbf{H}_{j}=-\mathbf{I}_{2}$. The update formula (17a) with $\mathbf{M}_{i}=m_{i} \mathbf{I}_{2}$, $\mathbf{M}_{j}=m_{j} \mathbf{I}_{2}$ results in

$$
\begin{aligned}
& \overline{\mathbf{M}}_{i}=\mathbf{M}_{i}+\left(\overline{\mathbf{M}}_{j}-\overline{\mathbf{M}}_{j}\left(\begin{array}{l}
0 \\
1
\end{array}\right)\left(\left(\begin{array}{lll}
0 & 1
\end{array}\right) \overline{\mathbf{M}}_{j}\left(\begin{array}{l}
0 \\
1
\end{array}\right)\right)^{+}\left(\begin{array}{lll}
0 & 1
\end{array}\right) \overline{\mathbf{M}}_{j}\right) \\
& =\left(\begin{array}{cc}
m_{i}+m_{j} & 0 \\
0 & m_{i}
\end{array}\right) \text {. }
\end{aligned}
$$

In (17b), we have $\overline{\boldsymbol{f}}_{i}=\boldsymbol{f}_{i}$ since $\boldsymbol{k}_{j}^{(\mathrm{I})}=\boldsymbol{k}_{j}^{(\mathrm{II})}=\mathbf{0}$ and $\boldsymbol{f}_{j}=\mathbf{0}$. The equations of motion in absolute coordinates are

$$
\begin{aligned}
m_{i} \ddot{p}_{i, x}-\mu_{j, x} & =-d_{i, x} \dot{p}_{i, x}-c_{i, x} p_{i, x}, \\
m_{i} \ddot{p}_{i, y} & =-d_{i, y} \dot{p}_{i, y}-c_{i, y} p_{i, y}, \\
m_{j} \ddot{p}_{j, x}+\mu_{j, x} & =0, \\
m_{j} \ddot{p}_{j, y} & =0 .
\end{aligned}
$$

Because of $\ddot{p}_{j, x}(t)=\ddot{p}_{i, x}(t)$, we obtain $\mu_{j, x}(t)=-m_{j} \ddot{p}_{j, x}(t)=$ $-m_{j} \ddot{p}_{i, x}(t)$ and the equations of motion $(36 \mathrm{a}, \mathrm{b})$ get the form

$$
\begin{aligned}
\left(m_{i}+m_{j}\right) \ddot{p}_{i, x} & =-d_{i, x} \dot{p}_{i, x}-c_{i, x} p_{i, x}, \\
m_{i} \ddot{p}_{i, y} & =-d_{i, y} \dot{p}_{i, y}-c_{i, y} p_{i, y} .
\end{aligned}
$$

In the limit case $m_{i}=0$, a combined set of $r_{i}=1$ second order differential equation for $q_{i, x}(t)$ and $n_{i}-r_{i}=1$ first order differential equation for $q_{i, y}(t)$ is obtained, see also (28a) and (28b) with $\mathbf{J}_{i}=-\mathbf{I}_{2}, \mathbf{X}_{i}=\mathbf{I}_{2}$ and $\overline{\mathbf{\Lambda}}_{i}=m_{j}$ :

$$
\begin{aligned}
m_{j} \ddot{q}_{i, x} & =-d_{i, x} \dot{q}_{i, x}-c_{i, x} q_{i, x}, \\
0 & =-d_{i, y} \dot{q}_{i, y}-c_{i, y} q_{i, y} .
\end{aligned}
$$

For this second test problem, we have a scalar joint coordinate $\boldsymbol{q}_{j}=q_{j, y} \in \mathbb{R}$ that is again not explicitly defined but has to satisfy the linearly implicit second order differential equation

$m_{j}\left(\ddot{q}_{j, y}+\ddot{q}_{i, y}\right)=0$,

see $(25 b)$ and $(35 b)$.

\section{Conclusions}

Motivated by results from singular perturbation theory, multibody system models with bodies of small mass or nearly singular inertia terms are analysed considering the limit case of systems with rank-deficient body mass matrices. Replacing in a classical recursive multibody formalism the inverse of condensed body mass matrices by their MoorePenrose pseudo-inverse, the backward recursion phase may be adapted to the rank-deficient case.

The crucial point in the analysis is the evaluation of accelerations for successors of bodies with rank-deficient body mass matrix in the forward recursion phase. It was shown that bodies with rank-deficient body mass matrix may simply be skipped in forward recursion. The acceleration coordinates of joints leaving such bodies to one of its successors are not given in explicit form but satisfy a linearly implicit equation that may be handled conveniently by common general purpose DAE solvers.

For each body with rank-deficient body mass matrix, a mixed system of first and second order differential equations is obtained resulting in a first order DAE that has index 1 for multibody system models with appropriate damping terms in the force elements acting at the "zero mass" body. Further investigations will be necessary to analyse practical aspects of this index-1 assumption in more detail.

In future research, the basic framework that has been developed in the present paper for tree structured rigid multibody system models will be extended to flexible systems and to multibody system models with (holonomic) constraints. These additional results will provide the algorithmic basis for a reference implementation in industrial multibody system simulation software. 


\section{Appendix A}

\section{Useful results from numerical linear algebra}

To make the paper self-contained, we summarize in this appendix some basics of numerical linear algebra. A comprehensive discussion of these topics is given, e.g., by Golub and van Loan (1996).

Remark 1 Any symmetric, positive semi-definite matrix $\mathbf{A} \in \mathbb{R}^{m \times m}$ with $r:=\operatorname{rank} \mathbf{A} \leq m$ has an orthonormal basis of eigenvectors $\boldsymbol{x}_{1}, \boldsymbol{x}_{2}, \ldots, \boldsymbol{x}_{r}, \boldsymbol{x}_{r+1}, \ldots, \boldsymbol{x}_{m}$ corresponding to its eigenvalues $\lambda_{1} \geq \lambda_{2} \geq \ldots \geq \lambda_{r}>\lambda_{r+1}=\ldots=\lambda_{m}=0$. Summarizing the eigenvectors in the orthogonal matrix $\mathbf{X}:=\left[\boldsymbol{x}_{1}, \ldots, \boldsymbol{x}_{m}\right] \in \mathbb{R}^{m \times m}$, we get $\mathbf{A X}=\mathbf{X} \mathbf{\Lambda}$ with the diagonal matrix $\boldsymbol{\Lambda}:=\operatorname{diag}_{1 \leq k \leq m} \lambda_{k}$.

(a) With $\boldsymbol{\Lambda}^{1 / 2}:=\operatorname{diag}_{1 \leq k \leq m} \sqrt{\lambda_{k}}$, matrix $\mathbf{A}^{1 / 2}:=\mathbf{X} \mathbf{\Lambda}^{1 / 2} \mathbf{X}^{\top}$ is well defined and independent of the specific choice of orthogonal eigenvectors $\boldsymbol{x}_{1}, \ldots, \boldsymbol{x}_{m}$. Matrix $\mathbf{A}^{1 / 2}$ is symmetric, positive semi-definite and satisfies $\mathbf{A}^{1 / 2} \mathbf{A}^{1 / 2}=$ $\left(\mathbf{X} \Lambda^{1 / 2} \mathbf{X}^{\top}\right)\left(\mathbf{X} \boldsymbol{\Lambda}^{1 / 2} \mathbf{X}^{\top}\right)=\mathbf{X} \mathbf{\Lambda}^{1 / 2} \boldsymbol{\Lambda}^{1 / 2} \mathbf{X}^{\top}=\mathbf{X} \mathbf{\Lambda} \mathbf{X}^{\top}=\mathbf{A}$.

(b) With $\overline{\mathbf{\Lambda}}:=\operatorname{diag}_{1 \leq k \leq r} \lambda_{k} \in \mathbb{R}^{r \times r}$, the Moore-Penrose pseudo-inverse of $\mathbf{A}$ is given by

$\mathbf{A}^{+}=\mathbf{X} \boldsymbol{\Lambda}^{+} \mathbf{X}^{\top}$ with $\boldsymbol{\Lambda}^{+}:=\left(\begin{array}{cc}\overline{\boldsymbol{\Lambda}}^{-1} & \mathbf{0}_{r \times(m-r)} \\ \mathbf{0}_{(m-r) \times r} & \mathbf{0}_{(m-r) \times(m-r)}\end{array}\right)$.

It defines orthogonal projectors $\mathbf{A A}^{+}$and $\mathbf{I}_{m}-\mathbf{A}^{+} \mathbf{A}$ projecting on the range of $\mathbf{A}$ and on the null space of $\mathbf{A}$, respectively:

$\mathbf{A} \mathbf{A}^{+} \mathbf{A}=\mathbf{A},\left(\mathbf{I}_{m}-\mathbf{A} \mathbf{A}^{+}\right) \mathbf{A}=\mathbf{0}_{m \times m}, \quad \mathbf{A}\left(\mathbf{I}_{m}-\mathbf{A}^{+} \mathbf{A}\right)=\mathbf{0}_{m \times m}$.

If $\mathbf{A}$ is not only positive semi-definite, but even positive definite, the Moore-Penrose pseudo-inverse $\mathbf{A}^{+}$coincides with the classical inverse $\mathbf{A}^{-1}$ since $\mathbf{A}$ is non-singular and $r=\operatorname{rank} \mathbf{A}=m$ in that case, $\boldsymbol{\Lambda}^{+}=\boldsymbol{\Lambda}^{-1}$.

Lemma 1 Consider a symmetric, positive semi-definite matrix $\mathbf{M} \in \mathbb{R}^{d \times d}$, (square) matrices $\mathbf{H}, \mathbf{K} \in \mathbb{R}^{d \times d}$ of the same format and a (rectangular) matrix $\mathbf{J} \in \mathbb{R}^{d \times n}$ with $0<n \leq d$. If $\mathbf{K}$ is non-singular then matrix

\section{$\mathbf{K}^{-\top} \mathbf{H}^{\top}\left(\mathbf{M}-\mathbf{M J}\left(\mathbf{J}^{\top} \mathbf{M J}\right)^{+} \mathbf{J}^{\top} \mathbf{M}\right) \mathbf{H K}^{-1}$}

with $\mathbf{K}^{-\top}=\left(\mathbf{K}^{-1}\right)^{\top}$ is symmetric and positive semi-definite.

Proof The argument $\mathbf{J}^{\top} \mathbf{M J} \in \mathbb{R}^{n \times n}$ of the Moore-Penrose pseudo-inverse may be written as $\mathbf{J}^{\top} \mathbf{M J}=\mathbf{C}^{\top} \mathbf{C}$ with $\mathbf{C}:=\mathbf{M}^{1 / 2} \mathbf{J} \in \mathbb{R}^{d \times n}$. The singular value decomposition of $\mathbf{C}$ has the form $\mathbf{C}=\mathbf{P} \boldsymbol{\Sigma} \mathbf{Q}^{\top}$ with orthogonal matrices $\mathbf{P} \in \mathbb{R}^{d \times d}$, $\mathbf{Q} \in \mathbb{R}^{n \times n}$ and the $(d \times n)$-matrix

$\boldsymbol{\Sigma}=\left(\begin{array}{c}\boldsymbol{\Sigma}_{0} \\ \mathbf{0}_{(d-n) \times n}\end{array}\right)$ with $\boldsymbol{\Sigma}_{0}=\left(\begin{array}{cc}\overline{\boldsymbol{\Sigma}}_{0} & \mathbf{0}_{r \times(n-r)} \\ \mathbf{0}_{(n-r) \times r} & \mathbf{0}_{(n-r) \times(n-r)}\end{array}\right) \in \mathbb{R}^{n \times n}$,
$\overline{\boldsymbol{\Sigma}}_{0}:=\operatorname{diag}_{1 \leq k \leq r} \sigma_{k}$, that summarizes the positive singular values $\sigma_{1} \geq \sigma_{2} \geq \ldots \geq \sigma_{r}>0$ of matrix $\mathbf{C}$. Here, $r \leq n$ denotes the rank of $\mathbf{C}$ and the remaining singular values vanish identically: $\sigma_{r+1}=\ldots=\sigma_{n}=0$.

Applying (A1) to

$\mathbf{A}:=\mathbf{C}^{\top} \mathbf{C}=\left(\mathbf{P} \boldsymbol{\Sigma} \mathbf{Q}^{\top}\right)^{\top}\left(\mathbf{P} \boldsymbol{\Sigma} \mathbf{Q}^{\top}\right)=\mathbf{Q} \boldsymbol{\Sigma}^{\top} \boldsymbol{\Sigma} \mathbf{Q}^{\top}$,

we get $m=n, \mathbf{X}=\mathbf{Q}$ and $\boldsymbol{\Lambda}=\boldsymbol{\Sigma}^{\top} \boldsymbol{\Sigma}=\boldsymbol{\Sigma}_{0}^{\top} \boldsymbol{\Sigma}_{0}$, i.e., $\overline{\boldsymbol{\Lambda}}=\overline{\boldsymbol{\Sigma}}_{0}^{\top} \overline{\boldsymbol{\Sigma}}_{0}$.

The assertion of the Lemma follows from

$$
\begin{aligned}
& \mathbf{K}^{-\top} \mathbf{H}^{\top}\left(\mathbf{M}-\mathbf{M J} \mathbf{J}\left(\mathbf{J}^{\top} \mathbf{M J}\right)^{+} \mathbf{J}^{\top} \mathbf{M}\right) \mathbf{H} \mathbf{K}^{-1} \\
& \quad=\left(\mathbf{M}^{1 / 2} \mathbf{H K}^{-1}\right)^{\top}\left(\mathbf{I}_{d}-\mathbf{C}\left(\mathbf{C}^{\top} \mathbf{C}\right)^{+} \mathbf{C}^{\top}\right)\left(\mathbf{M}^{1 / 2} \mathbf{H K}^{-1}\right)
\end{aligned}
$$

and

$$
\begin{aligned}
\mathbf{I}_{d} & -\mathbf{C}\left(\mathbf{C}^{\top} \mathbf{C}\right)^{+} \mathbf{C}^{\top} \\
& =\mathbf{P}(\mathbf{I}_{d}-\left(\begin{array}{c}
\boldsymbol{\Sigma}_{0} \\
\mathbf{0}
\end{array}\right) \underbrace{\mathbf{Q}^{\top} \mathbf{Q}}_{=\mathbf{I}_{n}}\left(\boldsymbol{\Sigma}_{0}^{\top} \boldsymbol{\Sigma}_{0}\right)^{+} \underbrace{\mathbf{Q}^{\top} \mathbf{Q}}_{=\mathbf{I}_{n}}\left(\begin{array}{cc}
\boldsymbol{\Sigma}_{0}^{\top} & \mathbf{0}
\end{array}\right)) \mathbf{P}^{\top} \\
& =\mathbf{P}\left(\mathbf{I}_{d}-\left(\begin{array}{cc}
\mathbf{I}_{r} & \mathbf{0} \\
\mathbf{0} & \mathbf{0}
\end{array}\right)\right) \mathbf{P}^{\top}=\mathbf{P}\left(\begin{array}{cc}
\mathbf{0} & \mathbf{0} \\
\mathbf{0} & \mathbf{I}_{d-r}
\end{array}\right) \mathbf{P}^{\top},
\end{aligned}
$$

see (A1).

Lemma 2 For any matrix $\mathbf{C} \in \mathbb{R}^{d \times n}$ with $0<n \leq d$ the Moore-Penrose pseudo-inverse of $\left(\mathbf{C}^{\top} \mathbf{C}\right) \in \mathbb{R}^{n \times n}$ satisfies

(a) $\mathbf{C}\left(\mathbf{I}_{n}-\left(\mathbf{C}^{\top} \mathbf{C}\right)^{+}\left(\mathbf{C}^{\top} \mathbf{C}\right)\right)=\mathbf{0}_{d \times n}$,

(b) $\left(\mathbf{I}_{n}-\left(\mathbf{C}^{\top} \mathbf{C}\right)\left(\mathbf{C}^{\top} \mathbf{C}\right)^{+}\right) \mathbf{C}^{\top}=\mathbf{0}_{n \times d}$.

Proof (a) Eq. (A2) with $\mathbf{A}:=\mathbf{C}^{\top} \mathbf{C} \in \mathbb{R}^{n \times n}$ yields

$$
\begin{aligned}
& \left(\mathbf{C}^{\top} \mathbf{C}\right)\left(\mathbf{I}_{n}-\left(\mathbf{C}^{\top} \mathbf{C}\right)^{+}\left(\mathbf{C}^{\top} \mathbf{C}\right)\right) \\
& \quad=\mathbf{C}^{\top} \mathbf{C}-\left(\mathbf{C}^{\top} \mathbf{C}\right)\left(\mathbf{C}^{\top} \mathbf{C}\right)^{+}\left(\mathbf{C}^{\top} \mathbf{C}\right)=\mathbf{C}^{\top} \mathbf{C}-\mathbf{C}^{\top} \mathbf{C}=\mathbf{0}_{n \times n},
\end{aligned}
$$

i.e., $\quad \mathbf{C}^{\top} \mathbf{C} \boldsymbol{z}_{k}=\mathbf{0}_{n}$ for the column vectors $\boldsymbol{z}_{k} \in \mathbb{R}^{n}$, $(k=1, \ldots, n)$, of matrix $\mathbf{I}_{n}-\left(\mathbf{C}^{\top} \mathbf{C}\right)^{+}\left(\mathbf{C}^{\top} \mathbf{C}\right)$. Since $\mathbf{C}^{\top} \mathbf{C} \boldsymbol{z}_{k}=\mathbf{0}_{n}$ implies $\boldsymbol{z}_{k}^{\top} \mathbf{C}^{\top} \mathbf{C} \boldsymbol{z}_{k}=0$ and $\left\|\mathbf{C} \boldsymbol{z}_{k}\right\|_{2}^{2}=0$, we get $\mathbf{C} z_{k}=\mathbf{0}_{d},(k=1, \ldots, n)$, and see that all column vectors of $\mathbf{I}_{n}-\left(\mathbf{C}^{\top} \mathbf{C}\right)^{+}\left(\mathbf{C}^{\top} \mathbf{C}\right)$ belong to the null space of $\mathbf{C}$.

(b) Assertion (b) follows in the same way from

$$
\left(\mathbf{I}_{n}-\left(\mathbf{C}^{\top} \mathbf{C}\right)\left(\mathbf{C}^{\top} \mathbf{C}\right)^{+}\right)\left(\mathbf{C}^{\top} \mathbf{C}\right)=\mathbf{C}^{\top} \mathbf{C}-\mathbf{C}^{\top} \mathbf{C}=\mathbf{0}_{n \times n} .
$$

Lemma 3 Consider a finite index set $I \subset \mathbb{N}_{+}$and matrices $\mathbf{B}_{0} \in \mathbb{R}^{d \times d}, \mathbf{B}_{j} \in \mathbb{R}^{d \times d},(j \in I), \mathbf{J} \in \mathbb{R}^{d \times n}$ with $0<n \leq d$. Then, matrix $\mathbf{B}_{0}^{\top} \mathbf{B}_{0}+\sum_{j \in I} \mathbf{B}_{j}^{\top} \mathbf{B}_{j}$ is symmetric, positive semidefinite and

$$
\mathbf{C}:=\left(\mathbf{B}_{0}^{\top} \mathbf{B}_{0}+\sum_{j \in I} \mathbf{B}_{j}^{\top} \mathbf{B}_{j}\right)^{1 / 2} \mathbf{J} \in \mathbb{R}^{d \times n}
$$

is well-defined and satisfies

$\mathbf{B}_{j} \mathbf{J}\left(\mathbf{I}_{n}-\left(\mathbf{C}^{\top} \mathbf{C}\right)^{+}\left(\mathbf{C}^{\top} \mathbf{C}\right)\right)=\mathbf{0}_{d \times n},(j \in I)$. 
Proof For any vector $z \in \mathbb{R}^{n}$, we get

$\boldsymbol{z}^{\top}\left(\mathbf{B}_{0}^{\top} \mathbf{B}_{0}+\sum_{j \in I} \mathbf{B}_{j}^{\top} \mathbf{B}_{j}\right) \boldsymbol{z}=\left\|\mathbf{B}_{0} z\right\|_{2}^{2}+\sum_{j \in I}\left\|\mathbf{B}_{j} z\right\|_{2}^{2} \geq 0$,

i.e., the symmetric matrix $\mathbf{B}_{0}^{\top} \mathbf{B}_{0}+\sum_{j} \mathbf{B}_{j}^{\top} \mathbf{B}_{j}$ is positive semidefinite and matrix $\mathbf{C} \in \mathbb{R}^{d \times n}$ is well-defined.

From the proof of Lemma 2 we know that $\mathbf{C} \boldsymbol{z}_{k}=\mathbf{0}_{d}$ for all column vectors $\boldsymbol{z}_{k}$ of matrix $\mathbf{I}_{n}-\left(\mathbf{C}^{\top} \mathbf{C}\right)^{+}\left(\mathbf{C}^{\top} \mathbf{C}\right)$. Therefore,

$$
\begin{aligned}
0 & =\left(\mathbf{C} \boldsymbol{z}_{k}\right)^{\top}\left(\mathbf{C} \boldsymbol{z}_{k}\right)=\boldsymbol{z}_{k}^{\top}\left(\mathbf{C}^{\top} \mathbf{C}\right) \boldsymbol{z}_{k} \\
& =\boldsymbol{z}_{k}^{\top} \mathbf{J}^{\top}\left(\mathbf{B}_{0}^{\top} \mathbf{B}_{0}+\sum_{j} \mathbf{B}_{j}^{\top} \mathbf{B}_{j}\right) \mathbf{J} \boldsymbol{z}_{k} \\
& =\boldsymbol{z}_{k}^{\top} \mathbf{J}^{\top} \mathbf{B}_{0}^{\top} \mathbf{B}_{0} \mathbf{J} \boldsymbol{z}_{k}+\sum_{j} \boldsymbol{z}_{k}^{\top} \mathbf{J}^{\top} \mathbf{B}_{j}^{\top} \mathbf{B} j \mathbf{J} z_{k} \\
& =\left\|\mathbf{B}_{0} \mathbf{J} \boldsymbol{z}_{k}\right\|_{2}^{2}+\sum_{j}\left\|\mathbf{B}_{j} \mathbf{J} \boldsymbol{z}_{k}\right\|_{2}^{2} .
\end{aligned}
$$

This sum of non-negative numbers may vanish only, if $\left\|\mathbf{B}_{0} \mathbf{J} z_{k}\right\|_{2}=0$ and $\left\|\mathbf{B}_{j} \mathbf{J} z_{k}\right\|_{2}=0,(j \in I, k=1, \ldots, n)$. Therefore, $\mathbf{B}_{j} \mathbf{J} \boldsymbol{z}_{k}=\mathbf{0}_{d}$ for all $j \in I$ and all column vectors $\boldsymbol{z}_{k}$, $(k=1, \ldots, n)$, of matrix $\mathbf{I}_{n}-\left(\mathbf{C}^{\top} \mathbf{C}\right)^{+}\left(\mathbf{C}^{\top} \mathbf{C}\right)$.

Acknowledgements. This research on recursive multibody formalisms for tree structured systems is funded by the German Federal Ministry of Education and Research (BMBF grant 05M10NHB). It extends previous results for chain structured systems that were obtained in a project "Quasistatic methods for numerical analysis of flexible multibody systems" that was funded by the German Research Foundation (grant AR 243/2-1). The fruitful cooperation with Bernhard Burgermeister (SIMPACK AG), Steffen Weber (Martin Luther University Halle-Wittenberg) and Michael Valášek (CTU Prague) is gratefully acknowledged. Figures 1, 2 and 3 were adapted from Arnold et al. (2010) and were provided by Bernhard Burgermeister and Steffen Weber, respectively.

Edited by: A. Tasora

Reviewed by: C. Führer and one anonymous referee

\section{References}

Arnold, M., Burgermeister, B., and Weber, S.: Improved time integration of multibody system models using methods from singular perturbation theory, in: Proc. of The 1st Joint International Conference on Multibody System Dynamics, 25-27 May 2010, Lappeenranta, Finland, 2010.
Brandl, H., Johanni, R., and Otter, M.: A very efficient algorithm for the simulation of robots and similar multibody systems without inversion of the mass matrix, in: Theory of Robots, edited by: Kopacek, P., Troch, I., and Desoyer, K., 95-100, Pergamon Press, Oxford, 1988.

Brenan, K., Campbell, S., and Petzold, L.: Numerical solution of initial-value problems in differential-algebraic equations, SIAM, Philadelphia, 2nd Edn., 1996.

Burgermeister, B., Arnold, M., and Eichberger, A.: Smooth velocity approximation for constrained systems in real-time simulation, Multibody Syst. Dyn., 26, 1-14, doi:10.1007/s11044-011-9243$1,2011$.

Eich-Soellner, E. and Führer, C.: Numerical Methods in Multibody Dynamics, Teubner-Verlag, Stuttgart, 1998.

Eichberger, A.: Transputer-based multibody system dynamic simulation: Part I. The residual algorithm - a modified inverse dynamic formulation, Mech. Struct. Mach., 22, 211-237, 1994.

Eichberger, A. and Rulka, W.: Process save reduction by macro joint approach: The key to real time and efficient vehicle simulation, Vehicle Syst. Dyn., 41, 401-413, 2004.

Golub, G. and van Loan, C.: Matrix Computations, The Johns Hopkins University Press, Baltimore London, 3rd Edn., 1996.

Hager, C. and Wohlmuth, B.: Analysis of a space-time discretization for dynamic elasticity problems based on mass-free surface elements, SIAM J. Numer. Anal., 47, 1863-1885, 2009.

Hairer, E. and Wanner, G.: Solving Ordinary Differential Equations. II. Stiff and Differential-Algebraic Problems, Springer-Verlag, Berlin Heidelberg New York, 2nd Edn., 1996.

Lubich, C.: Integration of stiff mechanical systems by Runge-Kutta methods, Z. Angew. Math. Phys., 44, 1022-1053, 1993.

Lubich, C., Nowak, U., Pöhle, U., and Engstler, C.: MEXX - Numerical software for the integration of constrained mechanical multibody systems, Tech. Rep. SC 92-12, ZIB Berlin, 1992.

Schiehlen, W.: Multibody system dynamics: roots and perspectives, Multibody Syst. Dyn., 1, 149-188, 1997.

Schönen, R.: Strukturdynamische Mehrkörpersimulation des Verbrennungsmotors mit elastohydrodynamischer Grundlagerkopplung, Fortschrittsberichte Strukturanalyse und Tribologie, Band 15, Kassel University Press, Kassel, 2003.

Stumpp, T.: Asymptotic expansions and attractive invariant manifolds of strongly damped mechanical systems, ZAMM - Z. Angew. Math. Mech., 88, 630-643, 2008.

Udwadia, F. and Phohomsiri, P.: Explicit equations of motion for constrained mechanical systems with singular mass matrices and applications to multi-body dynamics, Proc. R. Soc. A, 462, 2097-2117, doi:10.1098/rspa.2006.1662, 2006.

Weber, S., Arnold, M., and Valášek, M.: Quasistatic approximations for stiff second-order differential equations, Appl. Numer. Math., 62, 1579-1590, doi:10.1016/j.apnum.2012.06.030, 2012. 